\title{
Engineering Highway Hierarchies
}

\author{
Peter Sanders and Dominik Schultes \\ Universität Karlsruhe (TH), 76128 Karlsruhe, Germany, \{sanders, schultes\}@ira.uka.de
}

\begin{abstract}
Highway hierarchies exploit hierarchical properties inherent in real-world road networks to allow fast and exact point-to-point shortest-path queries. A fast preprocessing routine iteratively performs two steps: first, it removes edges that only appear on shortest paths close to source or target; second, it identifies low-degree nodes and bypasses them by introducing shortcut edges. The resulting hierarchy of highway networks is then used in a Dijkstra-like bidirectional query algorithm to considerably reduce the search space size without losing exactness. The crucial fact is that 'far away' from source and target it is sufficient to consider only high-level edges.

Various experiments with real-world road networks confirm the performance of our approach. On a $2.0 \mathrm{GHz}$ machine, preprocessing the network of Western Europe, which consists of about 18 million nodes, takes 13 minutes and yields 48 bytes of additional data per node. Then, random queries take $0.61 \mathrm{~ms}$ on average. If we are willing to accept slower query times $(1.10 \mathrm{~ms})$, the memory usage can be decreased to 17 bytes per node. We can guarantee that at most $0.014 \%$ of all nodes are visited during any query. Results for US road networks are similar.

Highway hierarchies can be combined with goal-directed search, they can be extended to answer manyto-many queries, and they are a crucial ingredient for other speedup techniques, namely for transit-node routing and highway-node routing.
\end{abstract}

\section{Introduction}

Computing fastest routes in road networks from a given source to a given target location is one of the showpieces of real-world applications of algorithmics. Many people frequently use this functionality when planning trips with their cars. There are also many applications like logistic planning or traffic simulation that need to solve a huge number of shortest-path queries. In principle we could use Dijkstra's algorithm [1]. But for large road networks this would be far too slow. Therefore, there is considerable interest in speedup techniques for route planning. Most approaches, including ours, assume that the road network is static, i.e., it does not change very often. Then, we can allow some preprocessing that generates auxiliary data that can be used to accelerate all subsequent queries. The preprocessing should be sufficiently fast to deal even with very large road networks, the auxiliary data should occupy only a moderate amount of space, and the queries should be as fast as possible.

\subsection{Related Work}

A detailed overview on shortest-path speedup techniques can be found in [2].

Bidirectional Search. A classical technique is bidirectional search which simultaneously searches forward from the source and backwards from the target until the search frontiers meet. Many more advanced speedup techniques use bidirectional search as an ingredient.

Goal Direction. Road networks allow effective goal-directed search using $\mathbf{A}^{*}$ search [3]: lower bounds define a vertex potential that directs search towards the target. This approach was recently shown to be very effective if lower bounds are computed using precomputed shortest-path distances to a carefully selected set of about 20 Landmark nodes [4,5] using the Triangle inequality $(A L T)$.

The Precomputed Cluster Distances (PCD) technique [6] also uses precomputed distances for goal-directed search, yielding speedups comparable to ALT, but using less space. 
The network is partitioned into clusters and the shortest connection between any pair of clusters is precomputed. Then, during a query, upper and lower bounds can be derived that can be used to prune the search.

Another goal-directed approach is to precompute for each edge 'signposts' that support the decision whether the target can possibly be reached on a shortest path via this edge. During a query only promising edges have to be considered. Various instantiations of this general idea have been presented [7-13]. While these methods exhibit good query performance, preprocessing times are quite large and so far no experimental results for the largest publicly available road networks have been published.

Separators. Perhaps the most well known property of road networks is that they are almost planar, i.e, techniques developed for planar graphs will often also work for road networks. Queries accurate within a factor $(1+\epsilon)$ can be answered in near constant time using $O((n \log n) / \epsilon)$ space and preprocessing time [14]. Recently, this approach has been efficiently implemented and experimentally evaluated on a road network with one million nodes [15]. While the query times are very good (less than $20 \mu \mathrm{s}$ for $\epsilon=0.01$ ), the preprocessing time and space consumption are quite high (2.5 hours and $2 \mathrm{~GB}$, respectively). Using $O\left(n \log ^{3} n\right)$ space and preprocessing time, query time $O(\sqrt{n} \log n)$ can be achieved [16] for directed planar graphs without negative cycles.

Another previous approach is the separator-based multi-level method [7,17]. The idea is to use a set of nodes $V_{1}$ whose removal partitions the graph $G=G_{0}$ into small components. Now consider the overlay graph $G_{1}=\left(V_{1}, E_{1}\right)$ where edges in $E_{1}$ are shortcuts corresponding to shortest paths in $G$ that do not have inner nodes that belong to $V_{1}$. Routing can now be restricted to $G_{1}$ and the components containing $s$ and $t$ respectively. This process can be iterated yielding a multi-level method. A limitation of this approach is that the graphs at higher levels become much more dense than the input graphs, thus limiting the benefits gained from the hierarchy. Also, computing small separators can become quite costly for large graphs.

Reach-Based Routing / REAL. Let $R(v):=\max _{s, t \in V} R_{s t}(v)$ denote the reach of node $v$ where $R_{s t}(v):=\min (d(s, v), d(v, t))$. Gutman [18] observed that a shortest-path search can be stopped at nodes with a reach too small to get to source or target from there. Goldberg et al. $[19,20]$ have considerably strengthened this approach by introducing various improvements, in particular a combination with ALT, yielding the REAL algorithm. Its query performance is similar to our highway hierarchies, while the preprocessing times are usually worse; a comparison can be found in Section 6.7.

Heuristics. In the last decades, commercial navigation systems were developed which had to handle ever more detailed descriptions of road networks on rather low-powered processors. Vendors resolved to heuristics still used today that do not give any performance guarantees: $A^{*}$ search with estimates on the distance to the target rather than lower bounds or heuristic hierarchical approaches [21,22].

\subsection{Our Contributions}

Our exact highway hierarchies (first published in [23,24]) are inspired by heuristic hierarchical approaches. It is a bidirectional technique. While the search is inside some local area around source or target, all roads of the network are considered. Outside these areas, however, the search is restricted to 'important' roads. This general idea can be iterated and 
applied to a hierarchy consisting of several levels. The crucial point is the definition of 'important streets'. In previous heuristic variants, this definition is based on a classification of the streets according to their type (motorway, national road, regional road, ...). Such a classification requires manual tuning of the data and a delicate trade-off between speed and suboptimality of the computed routes. In our exact variant, however, nodes and edges are classified fully automatically in a preprocessing step in such a way that all shortest paths are preserved. By this means, we win not only exactness, but also greater speed since we can build high-performance hierarchies consisting of many levels without worrying about the quality of the results.

In the preprocessing phase, we alternate between two procedures: edge reduction and node reduction. Edge reduction removes non-highway edges, i.e., edges that only appear on shortest paths close to source or target. More specifically, every node $v$ has a neighbourhood radius $r(v)$ we are free to choose. An edge $(u, v)$ is a highway edge if it belongs to some shortest path from a node $s$ to a node $t$ such that $(u, v)$ is neither fully contained in the neighbourhood of $s$ nor in the neighbourhood of $t$, i.e., $d(s, v)>r(s)$ and $d(u, t)>r(t)$. In all our experiments, neighbourhood radii are chosen such that each neighbourhood contains a certain number $H$ of nodes. $H$ is a tuning parameter that can be used to control the rate at which the network shrinks.

Node reduction (also called contraction) removes low degree nodes by bypassing them with newly introduced shortcut edges. In particular, all nodes of degree one and two are removed by this process.

The query algorithm is very similar to bidirectional Dijkstra search with the difference that certain edges need not be expanded when the search is sufficiently far from source or target. Highway hierarchies are the first speedup technique that was able handle the largest available road networks giving query times measured in milliseconds. There are two main reasons for this success: Under the above reduction routines, the road network shrinks in a geometric fashion from level to level and remains sparse and near planar, i.e., levels of the highway hierarchy are in some sense self similar. The other key property is that preprocessing can be done using limited local searches starting from each node. Preprocessing is also the most nontrivial aspect of highway hierarchies. In particular, long edges (e.g. longdistance ferry connections) make simple minded approaches far too slow. Instead we use fast heuristics that compute a superset of the set of highway edges.

Some further optimisations allow to drop the average query times below one millisecond on a $2.0 \mathrm{GHz}$ machine-even for a road network with more than 30 million nodes. One of these optimisations is an all-pairs distance table that we precompute for the topmost level $L$ so that forward and backward search can be stopped as soon as all entrance points to level $L$ have been found. Then, the remaining gap can be bridged by performing a moderate number of simple table lookups.

We cannot give a general worst-case bound better than Dijkstra's. So far, this drawback applies to all other exact speedup techniques, where an implementation is available, as well. However, in contrast to most of them, we can provide per-instance worst-case guarantees, i.e., for a given graph, we can determine an upper bound for the search space size of any possible point-to-point query performing only a linear number of unidirectional highway queries.

\subsection{Subsequent Work}

Various other speedup techniques were inspired by our highway hierarchies or even use them as their starting point. Goldberg et al. adopted the introduction of shortcuts in order to im- 
prove both preprocessing and query times of the REAL algorithm. There is a many-to-many variant [25] and a combination with ALT [26]. Furthermore, the fastest implementation of transit-node routing [27,28] allowing query times of a few microseconds is also based on our highway hierarchies. The same applies to highway-node routing [29], a very recent approach that can be used to handle dynamic scenarios, traffic jams for example. An alternative, heuristic approach to dealing with dynamic scenarios, which is based on highway hierarchies as well, has been developed by Nannicini et al. [30].

\subsection{Outline}

After beginning with some preliminaries in Section 2, we formally define the highway hierarchy of a given graph in Section 3. Then, Section 4 deals with both procedures of the preprocessing phase, the edge reduction (i.e., the construction of a highway network) and the node reduction (i.e., the contraction of a highway network). The basic query algorithm is introduced in Section 5. Furthermore, several optimisations are presented and some advanced topics, like outputting complete path descriptions and dealing with turning restrictions, are discussed. In Section 6, we present a wide range of experimental results, dealing with various real-world road networks, parameter settings, and scenarios of application. We do not only give average query times, but also a detailed analysis of queries with different degrees of difficulty, per-instance worst-case upper bounds, and comparisons to other speedup techniques.

\section{Preliminaries}

Graphs and Paths. We expect a directed graph $G=(V, E)$ with $n$ nodes and $m$ edges $(u, v)$ with nonnegative weights $w(u, v)$ as input. The length $w(P)$ of a path $P$ is the sum of the weights of the edges that belong to $P . P^{*}=\langle s, \ldots, t\rangle$ is a shortest path if there is no path $P^{\prime}$ from $s$ to $t$ such that $w\left(P^{\prime}\right)<w\left(P^{*}\right)$. The distance $d(s, t)$ between $s$ and $t$ is the length of a shortest path from $s$ to $t$ or $\infty$ if there is no path from $s$ to $t$. If $P=$ $\left\langle s, \ldots, s^{\prime}, u_{1}, u_{2}, \ldots, u_{k}, t^{\prime}, \ldots, t\right\rangle$ is a path from $s$ to $t$, then $\left.P\right|_{s^{\prime} \rightarrow t^{\prime}}=\left\langle s^{\prime}, u_{1}, u_{2}, \ldots, u_{k}, t^{\prime}\right\rangle$ denotes the subpath of $P$ from $s^{\prime}$ to $t^{\prime}$. We use $u \prec_{P} v$ to denote that a node $u$ precedes ${ }^{1}$ a node $v$ on a path $P=\langle\ldots, u, \ldots, v, \ldots\rangle$; we just write $u \prec v$ if the path $P$ that is referred to is clear from the context.

Dijkstra's Algorithm. Dijkstra's algorithm [1] can be used to solve the single-source shortestpath (SSSP) problem, i.e., to compute the shortest paths from a single source node $s$ to all other nodes in a given graph. It is covered by virtually any textbook on algorithms, e.g. [31, 32], so that we confine ourselves to introducing our terminology: Starting with the source node $s$ as root, Dijkstra's algorithm grows a shortest-path tree that contains shortest paths from $s$ to all other nodes. During this process, each node of the graph is unreached, reached, or settled. A node that already belongs to the tree is settled. If a node $u$ is settled, a shortest path $P^{*}$ from $s$ to $u$ has been found and the distance $d(s, u)=w\left(P^{*}\right)$ is known. A node that is adjacent to a settled node is reached. Note that a settled node is also reached. If a node $u$ is reached, a path $P$ from $s$ to $u$, which might not be the shortest one, has been found and a tentative distance $\delta(u)=w(P)$ is known. A node $u$ that is not reached is unreached; for such a node, we have $\delta(u)=\infty$.

In case that the shortest paths in a graph are not unique, Dijkstra's algorithm can be easily modified to determine all shortest paths between $s$ and any node $u \in V$. This means that not a shortest-path tree is grown, but a shortest-path directed acyclic graph (DAG).

\footnotetext{
${ }^{1}$ This does not necessarily mean that $u$ is the direct predecessor of $v$.
} 
A bidirectional version of Dijkstra's algorithm can be used to find a shortest path from a given node $s$ to a given node $t$. Two Dijkstra searches are executed in parallel: one searches from the source node $s$ in the original graph $G=(V, E)$, also called forward graph and denoted as $\vec{G}=(V, \vec{E})$; another searches from the target node $t$ backwards, i.e., it searches in the reverse graph $\overleftarrow{G}=(V, \overleftarrow{E}), \overleftarrow{E}:=\{(v, u) \mid(u, v) \in E\}$. The reverse graph $\overleftarrow{G}$ is also called backward graph. When both search scopes meet, a shortest path from $s$ to $t$ has been found.

\section{Highway Hierarchy}

A highway hierarchy of a graph $G$ consists of several levels $G_{0}, G_{1}, G_{2}, \ldots, G_{L}$, where the number of levels $L+1$ is given. We will provide an inductive definition of the levels:

- Base case $\left(G_{0}^{\prime}, G_{0}\right)$ : level $0\left(G_{0}=\left(V_{0}, E_{0}\right)\right)$ corresponds to the original graph $G$; furthermore, we define $G_{0}^{\prime}:=G_{0}$.

- First step $\left(G_{\ell}^{\prime} \rightarrow G_{\ell+1}, 0 \leq \ell<L\right)$ : for given neighbourhood radii, we will define the highway network $G_{\ell+1}$ of a graph $G_{\ell}^{\prime}$.

- Second step $\left(G_{\ell} \rightarrow G_{\ell}^{\prime}, 1 \leq \ell \leq L\right)$ : for a given set $B_{\ell} \subseteq V_{\ell}$ of bypassable nodes, we will define the core $G_{\ell}^{\prime}$ of level $\ell$.

First step (highway network). For each node $u$, we choose nonnegative neighbourhood radii $r_{\ell}(u)$ and $r_{\ell}^{\leftarrow}(u)$ for the forward and backward graph, respectively. To avoid some case distinctions, we set $r_{\ell}(u)$ and $r_{\ell}^{\leftarrow}(u)$ to infinity for $u \notin V_{\ell}^{\prime}$ (Radius Property R1) and for $\ell=L(\mathrm{R} 2)$. In all other cases, neighbourhood radii have to be $\neq \infty(\mathrm{R} 3)$.

The level- $\ell$ neighbourhood of a node $u \in V_{\ell}^{\prime}$ is $\mathcal{N}_{\ell}(u):=\left\{v \in V_{\ell}^{\prime} \mid d_{\ell}(u, v) \leq r_{\ell}(u)\right\}$ with respect to the forward graph and, analogously, $\mathcal{N}_{\ell}^{\leftarrow}(u):=\left\{v \in V_{\ell}^{\prime} \mid d_{\ell}^{\leftarrow}(u, v) \leq\right.$ $\left.r_{\ell}^{\leftarrow}(u)\right\}$ with respect to the backward graph, where $d_{\ell}(u, v)$ denotes the distance from $u$ to $v$ in the forward graph $G_{\ell}$ and $d_{\ell}^{\leftarrow}(u, v):=d_{\ell}(v, u)$ in the backward graph $\overleftarrow{G_{\ell}}$

The highway network $G_{\ell+1}=\left(V_{\ell+1}, E_{\ell+1}\right)$ of a graph $G_{\ell}^{\prime}$ is defined by the set $E_{\ell+1}$ of highway edges: an edge $(u, v) \in E_{\ell}^{\prime}$ belongs to $E_{\ell+1}$ iff there are nodes $s, t \in V_{\ell}^{\prime}$ such that the edge $(u, v)$ appears in some shortest path $\langle s, \ldots, u, v, \ldots, t\rangle$ from $s$ to $t$ in $G_{\ell}^{\prime}$ with the property that $v \notin \mathcal{N}_{\ell} \rightarrow(s)$ and $u \notin \mathcal{N}_{\ell}^{\leftarrow}(t)$. Figure 1 gives an example. The set $V_{\ell+1}$ is the maximal subset of $V_{\ell}^{\prime}$ such that $G_{\ell+1}$ contains no isolated nodes.

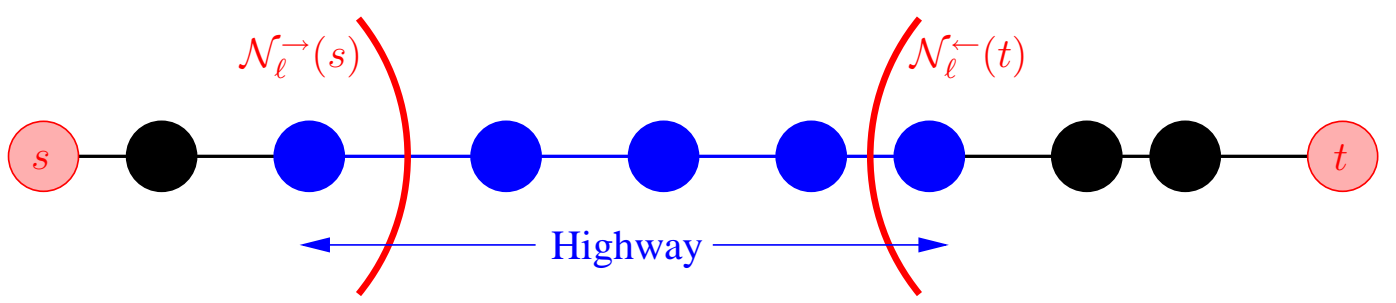

Fig. 1. A shortest path from a node $s$ to a node $t$. Edges that leave the neighbourhood of $s$ or $t$ and edges that are completely outside the neighbourhoods of $s$ and $t$ are highway edges.

Second step (core). For a given set $B_{\ell} \subseteq V_{\ell}$ of bypassable nodes, we define the set $S_{\ell}$ of shortcut edges that bypass the nodes in $B_{\ell}$ : for each path $P=\left\langle u, b_{1}, b_{2}, \ldots, b_{k}, v\right\rangle$ with $u, v \in V_{\ell} \backslash B_{\ell}$ and $b_{i} \in B_{\ell}, 1 \leq i \leq k$, the set $S_{\ell}$ contains an edge $(u, v)$ with $w(u, v)=w(P)$. The core $G_{\ell}^{\prime}=\left(V_{\ell}^{\prime}, E_{\ell}^{\prime}\right)$ of level $\ell$ is defined in the following way: 
$V_{\ell}^{\prime}:=V_{\ell} \backslash B_{\ell}$ and $E_{\ell}^{\prime}:=\left(E_{\ell} \cap\left(V_{\ell}^{\prime} \times V_{\ell}^{\prime}\right)\right) \cup S_{\ell}$. This definition is illustrated in Figure 2. Removing all core nodes from $G_{\ell}$ yields connected components of bypassed nodes.

The level $\ell(e)$ of an edge $e$ is $\max \left\{\ell \mid e \in E_{\ell} \cup S_{\ell}\right\}$. For an edge $(u, v)$, we usually write just $\ell(u, v)$ instead of $\ell((u, v))$. The highway hierarchy can be interpreted as a single graph $\mathcal{G}:=\left(V, E \cup \bigcup_{i=1}^{L} S_{i}\right)$ where each node and each edge has additional information on its membership in the various sets $V_{\ell}, V_{\ell}^{\prime}, B_{\ell}, E_{\ell}, E_{\ell}^{\prime}, S_{\ell}$.

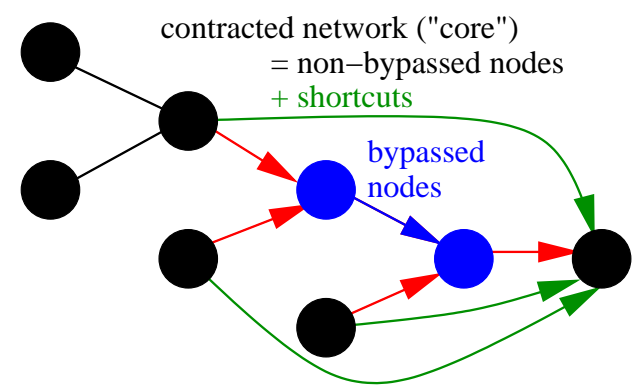

Fig. 2. The core of a highway network consists of the subgraph induced by the set of non-bypassed nodes and additional shortcut edges.

\section{Construction}

\subsection{Computing the Highway Network}

Neighbourhood Radii. Let us fix any rule that decides which element Dijkstra's algorithm removes from the priority queue in the case that there is more than one queued element with the smallest key. Then, during a Dijkstra search from a given node $u$, all nodes are settled in a fixed order. The Dijkstra rank $\operatorname{rk}_{u}(v)$ of a node $v$ is the rank of $v$ w.r.t. this order. $u$ has Dijkstra rank $\operatorname{rk}_{u}(u)=0$, the closest neighbour $v_{1}$ of $u$ has Dijkstra rank $\operatorname{rk}_{u}\left(v_{1}\right)=1$, and so on.

We suggest the following strategy to set the neighbourhood radii. For this paragraph, we interpret the graph $G_{\ell}^{\prime}$ as an undirected graph, i.e., a directed edge $(u, v)$ is interpreted as an undirected edge $\{u, v\}$ even if the edge $(v, u)$ does not exist in the directed graph. Let $d_{\ell}^{\leftrightarrow}(u, v)$ denote the distance between two nodes $u$ and $v$ in the undirected graph. For a given parameter $H_{\ell}$, for any node $u \in V_{\ell}^{\prime}$, we set $r_{\ell}(u):=r_{\ell}^{\leftarrow}(u):=d_{\ell}^{\leftrightarrow}(u, v)$, where $v$ is the node whose Dijkstra rank $\operatorname{rk}_{u}(v)$ (w.r.t. the undirected graph) is $H_{\ell}$. For any node $u \notin V_{\ell}^{\prime}$, we set $r_{\ell}(u):=r_{\ell}^{\leftarrow}(u):=\infty$ (to fulfil R1)

Originally, we wanted to apply the above strategy to the forward and backward graph one after the other in order to define the forward and backward radius, respectively. However, it turned out that using the same value for both forward and backward radius yields a similar good performance, but needs only half the memory.

Fast Construction: Outline. Given a graph $G_{\ell}^{\prime}$, we want to construct a highway network $G_{\ell+1}$. We start with an empty set of highway edges $E_{\ell+1}$. For each node $s_{0} \in V_{\ell}^{\prime}$, two phases are performed: the forward construction of a partial shortest-path DAG $B$ (containing all shortest paths from $s_{0}$ to any node $u \in B$ ) and the backward evaluation of $B$. The construction is done by an SSSP search from $s_{0}$; during the evaluation phase, paths from the leaves of $B$ to the root $s_{0}$ are traversed and for each edge on these paths, it is decided whether to add it to $E_{\ell+1}$ or not. The crucial part is the specification of an abort criterion for the SSSP search in order to restrict it to a 'local search'. 


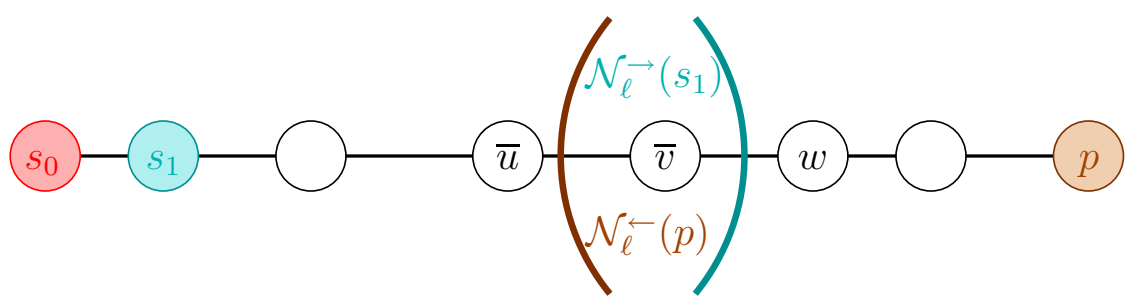

Fig. 3. Abort criterion.

Phase 1: Construction of a Partial Shortest-Path DAG. A Dijkstra search from $s_{0}$ is executed. In order to keep track of all shortest paths, for each node in the partial shortest-path DAG $B$, we manage a list of (tentative) parents: when an edge $(u, v)$ is relaxed such that $d_{\ell}\left(s_{0}, u\right)+w(u, v)=\delta(v)$, then $u$ is added to the list of tentative parents of $v$. During the search, a reached node is either in the state active or passive. The source node $s_{0}$ is active; each node that is reached for the first time (insert) and each reached node that is updated (decreaseKey) is set to active iff any of its tentative parents is active. When a node $p$ is settled, we consider all shortest paths $P^{\prime}$ from $s_{0}$ to $p$ as depicted in Figure 3. The state of $p$ is set to passive if

$\forall$ shortest paths $P^{\prime}=\left\langle s_{0}, \ldots, p\right\rangle$ :

$$
s_{1} \prec p \wedge p \notin \mathcal{N}_{\ell}^{\rightarrow}\left(s_{1}\right) \wedge s_{0} \notin \mathcal{N}_{\ell}^{\leftarrow}(p) \wedge\left|P^{\prime} \cap \mathcal{N}_{\ell} \rightarrow\left(s_{1}\right) \cap \mathcal{N}_{\ell}^{\leftarrow}(p)\right| \leq 1
$$

When no active unsettled node is left, the search is aborted and the growth of $B$ stops.

An example for Phase 1 of the construction is given in Figure 4. The intuitive reason for $s_{1}$ (which is the first successor of $s_{0}$ on the path $P^{\prime}$ ) to appear in the abort criterion is

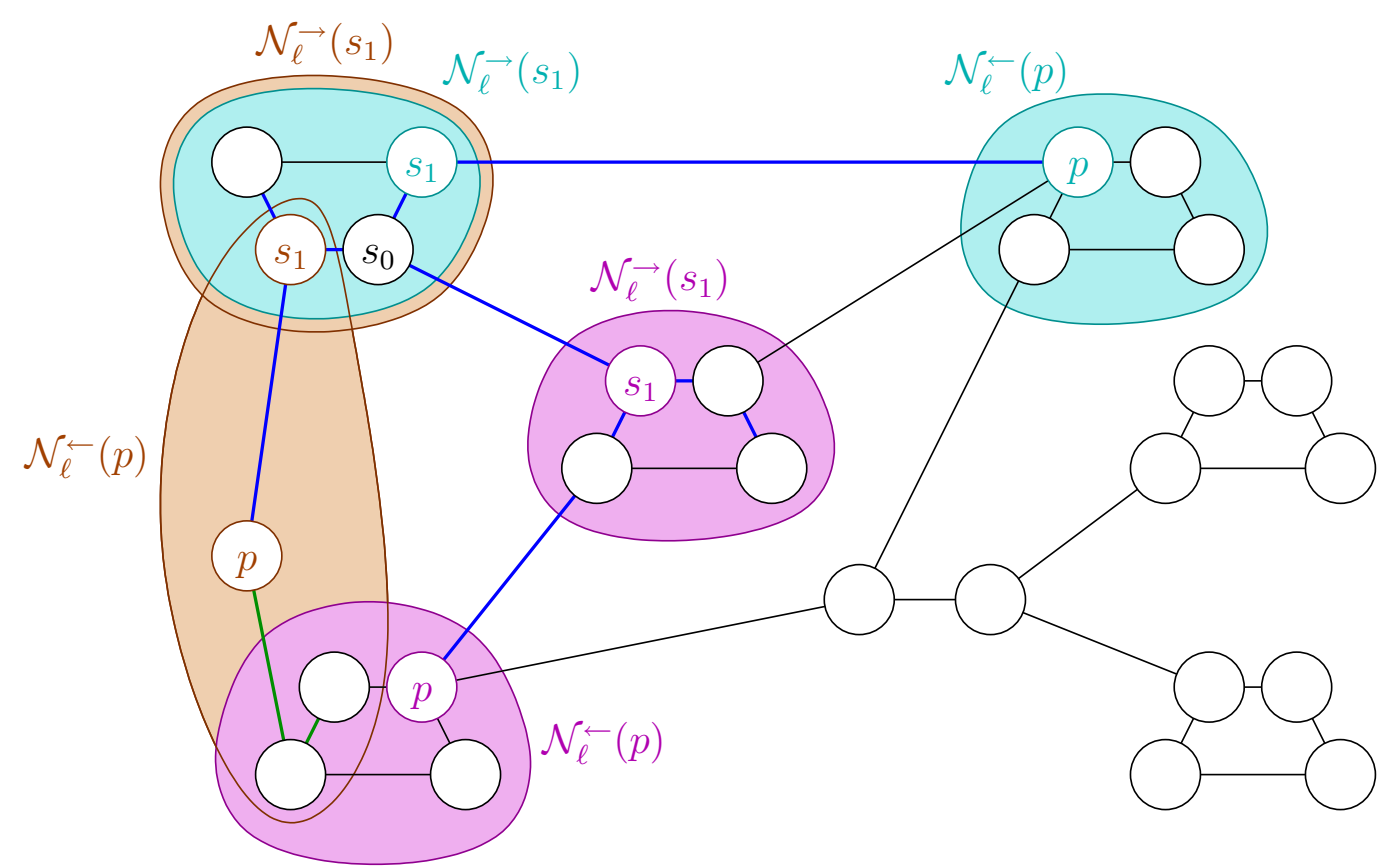

Fig. 4. An example of Phase 1 of the construction. The weight of an edge is the length of the line segment that represents the edge in this figure. The neighbourhood size $H_{\ell}$ is 3 . An SSSP search is performed from $s_{0}$. The abort criterion applies three times: the involved nodes $s_{1}$ and $p$ and the corresponding neighbourhoods are marked in cyan, magenta, and brown, respectively. In the brown case, the intersection of the concerned neighbourhoods contains exactly one element; in the other two cases, the intersections are empty. All edges that belong to $s_{0}$ 's partial shortest-path tree are coloured: edges that leave active nodes are blue, edges that leave passive nodes are green. 
the following: When we deactivate a node $p$ during the search from $s_{0}$, we decide to ignore everything that lies behind $p$. We are free to do this because the abort criterion ensures that $s_{1}$ can take 'responsibility' for the things that lie behind $p$, i.e., further important edges will be added during the search from $s_{1}$. (Of course, $s_{1}$ will refer a part of its 'responsibility' to its successor, and so on.)

Phase 2: Selection of the Highway Edges. During Phase 2, exactly all edges $(u, v)$ are added to $E_{\ell+1}$ that lie on paths $\left\langle s_{0}, \ldots, u, v, \ldots, p\right\rangle$ in the partial shortest-path DAG $B$ with the property that $v \notin \mathcal{N}_{\ell} \rightarrow\left(s_{0}\right)$ and $u \notin \mathcal{N}_{\ell}^{\leftarrow}(p)$. The example from Figure 4 is continued in Figure 5.

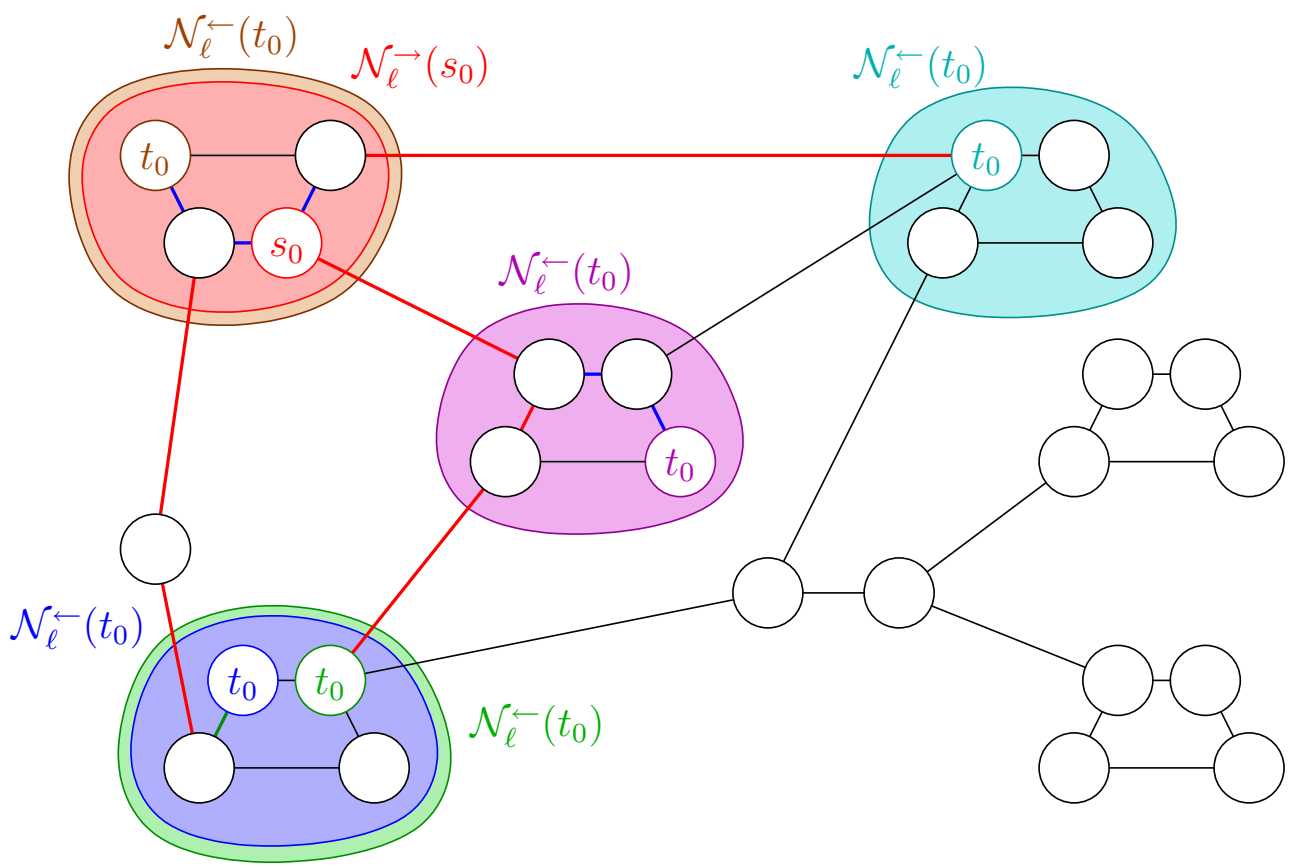

Fig. 5. An example of Phase 2 of the construction. $s_{0}$ 's partial shortest path tree has five leaves $t_{0}$, which are marked in different colours. The edges that are added to $E_{\ell+1}$ are highlighted.

Theorem 1. An edge $(u, v) \in E_{\ell}^{\prime}$ is added to $E_{\ell+1}$ by the construction algorithm iff it belongs to some shortest path $P=\langle s, \ldots, u, v, \ldots, t\rangle$ and $v \notin \mathcal{N}_{\ell} \rightarrow(s)$ and $u \notin \mathcal{N}_{\ell}^{\leftarrow}(t)$.

Proof. In this proof, we will refer to the following Neighbourhood Property N1 that follows directly from the neighbourhood definition: Consider a shortest path $\langle s, \ldots, u, \ldots, t\rangle$ in $G_{\ell}^{\prime}$. Then, $t \in \mathcal{N}_{\ell} \rightarrow(s)$ implies $u \in \mathcal{N}_{\ell} \rightarrow(s)$ and $s \in \mathcal{N}_{\ell}^{\leftarrow}(t)$ implies $u \in \mathcal{N}_{\ell}^{\leftarrow}(t)$.

$\Leftarrow)$ Consider the node $s_{0}$ on $\left.P\right|_{s \rightarrow u}$ such that $v \notin \mathcal{N}_{\ell}\left(s_{0}\right)$ and $d_{\ell}\left(s_{0}, v\right)$ is minimal. Such a node $s_{0}$ exists because the condition $v \notin \mathcal{N}_{\ell}\left(s_{0}\right)$ is always fulfilled for $s_{0}=s$. The direct successor of $s_{0}$ on $P$ is denoted by $s_{1}$. Note that $v \in \mathcal{N}_{\ell}\left(s_{1}\right)$ [*]. We show that the edge $(u, v)$ is added to $E_{\ell+1}$ when Phase 1 and 2 are executed from $s_{0}$. Due to the specification of Phase 2, it is sufficient to prove that after Phase 1 has been completed, the partial shortest-path DAG $B$ contains a node $\left.p \in P\right|_{s_{0} \rightarrow t}$ such that $v \preceq p$ and $u \notin \mathcal{N}_{\ell}^{\leftarrow}(p)$.

If $t \in B$, this statement is obviously fulfilled for $p:=t$ since $v \preceq t$ and $u \notin \mathcal{N}_{\ell}^{\leftarrow}(t)$. Otherwise $(t \notin B)$, the search is not continued from some node $t_{0} \prec t$ on $\left.P\right|_{s_{0} \rightarrow t}$. We can conclude that $t_{0}$ is passive because, otherwise, its successor on $\left.P\right|_{s_{0} \rightarrow t}$ would adopt its active state and the search would not be aborted at that time. Since $s_{0}$ is active and $t_{0}$ is passive, either $t_{0}$ or one of its ancestors must have been switched from active to passive. Let $p$ denote 
the first passive node on $\left.P\right|_{s_{0} \rightarrow t}=\left\langle s_{0}, s_{1}, \ldots, p, \ldots, t_{0}, \ldots, t\right\rangle$. Due to the definition of the abort condition, we have $s_{1} \prec p \wedge p \notin \mathcal{N}_{\ell} \rightarrow\left(s_{1}\right) \wedge s_{0} \notin \mathcal{N}_{\ell}^{\leftarrow}(p) \wedge\left|P^{\prime} \cap \mathcal{N}_{\ell} \rightarrow\left(s_{1}\right) \cap \mathcal{N}_{\ell}^{\leftarrow}(p)\right| \leq 1$ [**], where $P^{\prime}=\left.P\right|_{s_{0} \rightarrow p}$. The facts that $v \in \mathcal{N}_{\ell}\left(s_{1}\right)$ [see *] and $p \notin \mathcal{N}_{\ell}\left(s_{1}\right)$ [see **] imply $v \prec p$ due to N1. In order to obtain a contradiction, we assume $u \in \mathcal{N}_{\ell}^{\leftarrow}(p)$. Since $s_{0} \notin \mathcal{N}_{\ell}^{\leftarrow}(p)$ [see **], this implies $s_{0} \prec u$ by N1. Hence, $s_{1} \preceq u$. Because $v \in \mathcal{N}_{\ell} \rightarrow\left(s_{1}\right)$ [see *], we obtain $u \in \mathcal{N}_{\ell} \rightarrow\left(s_{1}\right)$ due to N1. Similarly, we get $v \in \mathcal{N}_{\ell}^{\leftarrow}(p)$ since $v \prec p$ and $u \in \mathcal{N}_{\ell}^{\leftarrow}(p)$. Thus, $\{u, v\} \subseteq P^{\prime} \cap \mathcal{N}_{\ell} \rightarrow\left(s_{1}\right) \cap \mathcal{N}_{\ell}^{\leftarrow}(p)$. Therefore, $\left|P^{\prime} \cap \mathcal{N}_{\ell} \rightarrow\left(s_{1}\right) \cap \mathcal{N}_{\ell}^{\leftarrow}(p)\right| \geq 2$, which is a contradiction to [**]. We can conclude that $u \notin \mathcal{N}_{\ell}^{\leftarrow}(p)$.

$\Rightarrow)$ Since each path $\left\langle s_{0}, \ldots, u, v, \ldots, p\right\rangle$ in $B$ is a shortest path, the claim follows directly from the specification of Phase 2.

Algorithmic Details: Phase 1. For an efficient implementation, we keep track of a border distance $b(x)$ and a reference distance $a(x)$ for each node $x$ in $B$. Along a path $P^{\prime}$ as depicted in Figure 3, we assign $b(x)$ the distance from the root to the border of the neighbourhood of $s_{1}$ as soon as $s_{1}$ is settled. This value is passed to all successors on the path, which allows to determine the first node $w$ outside $\mathcal{N}_{\ell} \rightarrow\left(s_{1}\right)$, i.e., its direct predecessor $\bar{v}$ is the last node inside $\mathcal{N}_{\ell} \rightarrow\left(s_{1}\right)$. In order to fulfil the abort condition, we have to make sure that $\bar{v}$ is the only node on $P^{\prime}$ within $\mathcal{N}_{\ell} \rightarrow\left(s_{1}\right) \cap \mathcal{N}_{\ell}^{\leftarrow}(p)$. Therefore, we want to check whether $\bar{v}$ 's direct predecessor $\bar{u}$ belongs to $\mathcal{N}_{\ell}^{\leftarrow}(p)$. To allow an easy check, we determine, store, and propagate the reference distance from $s_{0}$ to $\bar{u}$ as soon as $w$ is settled. Knowing the reference distance $d_{\ell}\left(s_{0}, \bar{u}\right)$, the current distance $d_{\ell}\left(s_{0}, p\right)$ and $p$ 's neighbourhood radius $r_{\ell}^{\leftarrow}(p)$, checking $\bar{u} \notin \mathcal{N}_{\ell}^{\leftarrow}(p)$ is then straightforward. If there are several shortest paths from $s_{0}$ to some node $x$, we determine appropriate maxima of the involved border and reference distances.

More formally, for any node $x$ in $B, \pi(x)$ denotes the set of parent nodes in $B$. To avoid some case distinctions, we set $\pi\left(s_{0}\right):=\left\{s_{0}\right\}$, i.e., the root is its own parent. For the root $s_{0}$, we set $b\left(s_{0}\right):=0$ and $a\left(s_{0}\right):=\infty$. For any other node $x \neq s_{0}$, we define $b^{\prime}(x):=$ $d_{\ell}\left(s_{0}, x\right)+r_{\ell}(x)$ if $s_{0} \in \pi(x)$, and 0, otherwise; $b(x):=\max \left(\left\{b^{\prime}(x)\right\} \cup\{b(y) \mid y \in \pi(x)\}\right)$; $a^{\prime}(x):=\max \{a(y) \mid y \in \pi(x)\}$; and $a(x):=\max \left\{d_{\ell}\left(s_{0}, u\right) \mid y \in \pi(x) \wedge u \in \pi(y)\right\}$ if $a^{\prime}(x)=\infty \wedge d_{\ell}\left(s_{0}, x\right)>b(x)$, and $a^{\prime}(x)$, otherwise.

Then, we can easily check the following abort criterion at a settled node $p$ :

$$
a(p)+r_{\ell}^{\leftarrow}(p)<d_{\ell}\left(s_{0}, p\right)
$$

Lemma 1. (2) implies (1).

Proof. We prove the contraposition " $\neg$ (1) implies $\neg$ (2)", i.e., we assume that there is some shortest path $P^{\prime}$ from $s_{0}$ to $p$ such that $p \preceq s_{1} \vee p \in \mathcal{N}_{\ell} \rightarrow\left(s_{1}\right) \vee s_{0} \in \mathcal{N}_{\ell}^{\leftarrow}(p) \vee \mid P^{\prime} \cap \mathcal{N}_{\ell} \rightarrow\left(s_{1}\right) \cap$ $\mathcal{N}_{\ell}^{\leftarrow}(p) \mid \geq 2$ and show that $a(p)+r_{\ell}^{\leftarrow}(p) \geq d_{\ell}\left(s_{0}, p\right)$.

Case 1: $p \preceq s_{1}$. If $p=s_{0}$, then $a(p)=\infty$, which yields $\neg(2)$. Otherwise $\left(p=s_{1}\right)$, $b(p) \geq d_{\ell}\left(s_{0}, p\right)+r_{\ell}(p), a^{\prime}(p)=\infty$, and $a(p)=a^{\prime}(p)$ since $d_{\ell}\left(s_{0}, p\right) \leq b(p)$, which implies $\neg(2)$.

Case 2: $s_{1} \prec p \wedge p \in \mathcal{N}_{\ell}\left(s_{1}\right)$. Due to N1 (see proof of Theorem 1), we have $\forall x, s_{1} \preceq$ $x \preceq p: x \in \mathcal{N}_{\ell} \rightarrow\left(s_{1}\right)$. Hence, $\forall x: d_{\ell}\left(s_{0}, x\right) \leq d_{\ell}\left(s_{0}, s_{1}\right)+r_{\ell}\left(s_{1}\right) \leq b(x)$. By an inductive proof, we can show that $a(p)=\infty$, which yields $\neg(2)$.

Case 3: $s_{1} \prec p \wedge p \notin \mathcal{N}_{\ell} \rightarrow\left(s_{1}\right) \wedge s_{0} \in \mathcal{N}_{\ell}^{\leftarrow}(p)$. We have $d_{\ell}\left(s_{0}, p\right) \leq r_{\ell}^{\leftarrow}(p)$, which directly implies $\neg(2)$.

Case 4: $s_{1} \prec p \wedge p \notin \mathcal{N}_{\ell} \rightarrow\left(s_{1}\right) \wedge s_{0} \notin \mathcal{N}_{\ell}^{\leftarrow}(p) \wedge\left|P^{\prime} \cap \mathcal{N}_{\ell}\left(s_{1}\right) \cap \mathcal{N}_{\ell}^{\leftarrow}(p)\right| \geq 2$. The assumption of Case 4 implies that there are two nodes $\bar{u}$ and $\bar{v}, s_{1} \preceq \bar{u} \prec \bar{v} \preceq p$, that belong to $P^{\prime} \cap \mathcal{N}_{\ell} \rightarrow\left(s_{1}\right) \cap \mathcal{N}_{\ell}^{\leftarrow}(p)$. If $a(p)=\infty$, we directly have $\neg$ (2). Otherwise, there has to be some node $w$ on $P^{\prime}$ such that $a^{\prime}(w)=\infty \wedge d_{\ell}\left(s_{0}, w\right)>b(w)$. Obviously, $w \neq s_{0}$. Consider 
such a node $w$ that maximises $d_{\ell}\left(s_{0}, w\right)$, i.e., for all nodes $x \succ w$ the above stated condition does not hold, which implies $a(x)=a^{\prime}(x) \geq a(w)$. In particular, $a(p) \geq a(w)$. We have $b(w) \geq d_{\ell}\left(s_{0}, s_{1}\right)+r_{\ell}\left(s_{1}\right)$. We can conclude that $d_{\ell}\left(s_{0}, w\right)>d_{\ell}\left(s_{0}, s_{1}\right)+r_{\ell}\left(s_{1}\right)$ and, thus, $w \notin \mathcal{N}_{\ell} \rightarrow\left(s_{1}\right)$. We obtain, by N1, $\bar{u} \prec \bar{v} \prec w$. Hence, $a(w) \geq d_{\ell}\left(s_{0}, \bar{u}\right)$, which implies $a(p) \geq d_{\ell}\left(s_{0}, \bar{u}\right)$. Furthermore, since $\bar{u} \in \mathcal{N}_{\ell}^{\leftarrow}(p)$, we have $r_{\ell}^{\leftarrow}(p) \geq d_{\ell}(\bar{u}, p)$. Adding up the last two inequalities yields $a(p)+r_{\ell}^{\leftarrow}(p) \geq d_{\ell}\left(s_{0}, p\right)$, which corresponds to $\neg$ (2).

Algorithmic Details: Phase 2. For a node $u \in B$, we define $\mathcal{B}(u):=\{u\} \cup\{v \mid v$ is a descendant of $u$ in $B\}$ and the slack $\Delta(u):=\min _{w \in \mathcal{B}(u)}\left(r_{\ell}^{\leftarrow}(w)-d_{\ell}(u, w)\right)$. For a leaf $b$, we have $\mathcal{B}(b)=\{b\}$ and $\Delta(b)=r_{\ell}^{\leftarrow}(b)$. The slack of an inner node $u$ can be computed using only the slacks of its children $C(u): \Delta(u)=\min \left(r_{\ell}^{\leftarrow}(u), \min _{c \in C(u)} \Delta_{c}(u)\right)$, where $\Delta_{c}(u):=\Delta(c)-d_{\ell}(u, c)$. This leads to an equivalent, recursive definition.

The tentative slacks $\widehat{\Delta}(u)$ of all nodes $u$ in $B$ are set to $r_{\ell}^{\leftarrow}(u)$. We process all nodes in the reverse order as they were settled. This guarantees that all descendants of some node $u$ have been processed before $u$ is processed. We can stop as soon as a node $u \in \mathcal{N}_{\ell}\left(s_{0}\right)$ is encountered. We maintain the invariant that the tentative slack $\widehat{\Delta}(u)$ of an element $u$ that is processed is equal to the actual slack $\Delta(u)$. When a node $u$ is processed, for each parent $p$ of $u$ in $B$, we perform the following steps: compute $\Delta_{u}(p)=\Delta(u)-d_{\ell}(p, u)$; if $\Delta_{u}(p)<0$, the edge $(p, u)$ is added to $E_{\ell+1}$; if $\Delta_{u}(p)<\widehat{\Delta}(p)$, the tentative slack $\widehat{\Delta}(p)$ is set to $\Delta_{u}(p)$. Figure 6 gives an example.

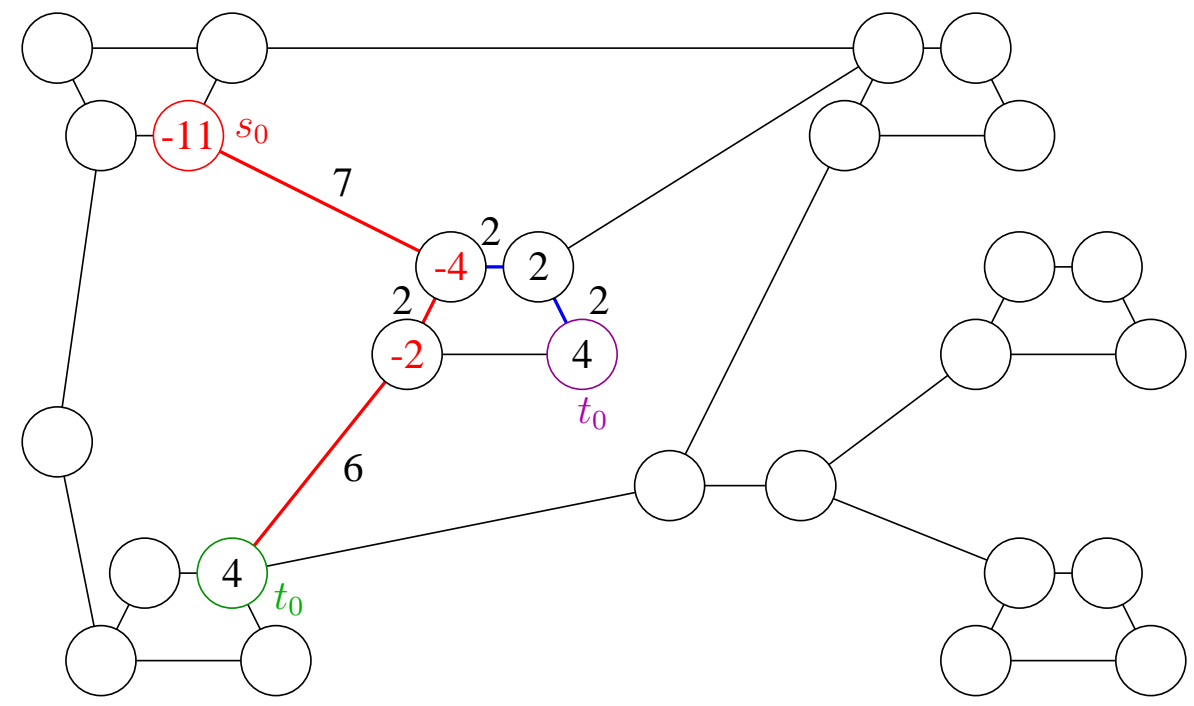

Fig. 6. An example of the slack-based method that realises Phase 2 of the construction. The process is shown only for a part of the tree. As before, the weight of an edge is the length of the line that represents the edge in this figure. For the sake of transparency, the (rounded) weights are given explicitly for the relevant edges. Furthermore, the slacks of the involved nodes are given. Edges that are added to $E_{\ell+1}$ are red, edges that are not added blue.

Theorem 2. An edge $(u, v)$ is added to $E_{\ell+1}$ by the slack-based method introduced above iff it lies on a path $\left\langle s_{0}, \ldots, u, v, \ldots, p\right\rangle$ in the partial shortest-path DAG B with the property that $v \notin \mathcal{N}_{\ell} \rightarrow\left(s_{0}\right)$ and $u \notin \mathcal{N}_{\ell}^{\leftarrow}(p)$.

Proof. $\Leftarrow)$ From the definition of the slack of a node, it follows that

$$
\Delta_{v}(u)=\Delta(v)-d_{\ell}(u, v) \leq r_{\ell}^{\leftarrow}(p)-d_{\ell}(v, p)-d_{\ell}(u, v)=r_{\ell}^{\leftarrow}(p)-d_{\ell}(u, p)<0
$$

because $u \notin \mathcal{N}_{\ell}^{\leftarrow}(p)$. Since $v \notin \mathcal{N}_{\ell} \rightarrow\left(s_{0}\right), v$ is processed at some point. Then, $\Delta_{v}(u)$ is computed and, since it is negative, the edge $(u, v)$ is added to $E_{\ell+1}$. 
$\Rightarrow$ ) Only edges that belong to a path in $B$ from $s_{0}$ to a node $p$ are considered. The condition $v \notin \mathcal{N}_{\ell} \rightarrow\left(s_{0}\right)$ is never violated because the traversal from the leaves to the root, and consequently, the addition of edges to $E_{\ell+1}$, is not continued when a node $v \in \mathcal{N}_{\ell}\left(s_{0}\right)$ is encountered. If an edge $(u, v)$ is added, the condition $\Delta_{v}(u)<0$ is fulfilled. Hence, $\Delta(u)=\min _{w \in \mathcal{B}(u)}\left(r_{\ell}^{\leftarrow}(w)-d_{\ell}(u, w)\right) \leq \Delta_{v}(u)<0$. Therefore, there is a node $p$ such that $d_{\ell}(u, p)>r_{\ell}^{\leftarrow}(p)$, i.e., $u \notin \mathcal{N}_{\ell}^{\leftarrow}(p)$.

Theorem 3. Let $V_{B}$ denote the set of nodes of $s_{0}$ 's partial shortest-path DAG B. Let $G_{B}=$ $\left(V_{B}, E_{B}\right)$ denote the subgraph of $G_{\ell}^{\prime}$ that is vertex induced by $V_{B}$. The complexity of Phase 1 and 2 started from $s_{0}$ is $T_{\text {Dijkstra }}\left(\left|G_{B}\right|\right)$.

Proof. The number of nodes of $G_{B}$ is denoted by $n^{\prime}$, the number of edges by $m^{\prime}$. The complexity of Phase 1 corresponds to the complexity of a SSSP search in $G_{B}$ started from $s_{0}$, i.e., $O\left(n^{\prime}+m^{\prime}\right)$ outside the priority queue plus $n^{\prime}$ insert and $n^{\prime}$ deleteMin operations plus at most $m^{\prime}$ decreaseKey operations. During Phase 2, each node and each edge is processed at most once, i.e., Phase 2 runs in $O\left(n^{\prime}+m^{\prime}\right)$.

Speeding Up the Highway Network Construction. Even a single active endpoint of a long edge (e.g., a long-distance ferry connection) can cause a large search space during construction, although most nodes of the search space might already be passive. To face this undesirable effect, we declare an active node $v$ to be a maverick if $d_{\ell}\left(s_{0}, v\right)>f \cdot r_{\ell}\left(s_{0}\right)$, where $f$ is a parameter. When all active nodes are mavericks, the search from passive nodes is no longer continued. This way, the construction process is accelerated and $E_{\ell+1}$ becomes a superset of the highway network. Hence, queries will be slower, but still compute exact shortest paths. The maverick factor $f$ enables us to adjust the trade-off between construction and query time.

\subsection{Computing the Core}

In order to obtain the core of a highway network, we contract it, which yields several advantages. The search space during the queries gets smaller since bypassed nodes are not touched and the construction process gets faster since the next iteration only deals with the nodes that have not been bypassed. Furthermore, a more effective contraction allows us to use smaller neighbourhood sizes without compromising the shrinking of the highway networks. This improves both construction and query times. However, bypassing nodes involves the creation of shortcuts, i.e., edges that represent the bypasses. Due to these shortcuts, the average degree of the graph is increased and the memory consumption grows. In particular, more edges have to be relaxed during the queries. Therefore, we have to carefully select nodes so that the benefits of bypassing them outweigh the drawbacks.

We give an iterative algorithm that combines the selection of the bypassable nodes $B_{\ell}$ with the creation of the corresponding shortcuts. We manage a stack that contains all nodes that have to be considered, initially all nodes from $V_{\ell}$. As long as the stack is not empty, we deal with the topmost node $u$. We check the bypassability criterion \#shortcuts $\leq c$. $\left(\operatorname{deg}_{\text {in }}(u)+\operatorname{deg}_{\text {out }}(u)\right)$, which compares the number of shortcuts that would be created when $u$ was bypassed with the sum of the in- and outdegree of $u$. The magnitude of the contraction is determined by the parameter $c$. If the criterion is fulfilled, the node is bypassed, i.e., it is added to $B_{\ell}$ and the appropriate shortcuts are created. Note that the creation of the shortcuts alters the degree of the corresponding endpoints so that bypassing one node can influence the bypassability criterion of another node. Therefore, all adjacent nodes that have been removed from the stack earlier, have not been bypassed, yet, and are bypassable now are pushed on the stack once again. 
Theorem 4. If $c<2,\left|E_{\ell}^{\prime}\right|$ is in $O\left(\left|V_{\ell}\right|+\left|E_{\ell}\right|\right)$.

Proof. If a node $u$ is bypassed, the number of edges in the (tentative) core is increased by $\mathcal{D}_{u}:=\#$ shortcuts $-\operatorname{deg}_{\text {in }}(u)-\operatorname{deg}_{\text {out }}(u)$. (We have to subtract $\operatorname{deg}_{\text {in }}(u)$ and $\operatorname{deg}_{\text {out }}(u)$ since the edges incident to $u$ no longer belong to the core.) Note that \#shortcuts $=\operatorname{deg}_{\text {in }}(u)$. $\operatorname{deg}_{\text {out }}(u)-\operatorname{deg}_{\leftrightarrow}(u)$, where $\operatorname{deg}_{\leftrightarrow}(u)$ denotes the number of adjacent nodes $v$ that are connected to $u$ by both an edge $(u, v)$ and an edge $(v, u)$. (We have to subtract $\operatorname{deg}_{\leftrightarrow}(u)$ to account for the fact that a 'shortcut' that would be a self-loop is not created.) We can conclude that $\mathcal{D}_{u} \leq \operatorname{deg}_{\text {in }}(u) \cdot \operatorname{deg}_{\text {out }}(u)-\operatorname{deg}_{\text {in }}(u)-\operatorname{deg}_{\text {out }}(u)$. If $\operatorname{deg}_{\text {in }}(u) \leq 1$ or $\operatorname{deg}_{\text {out }}(u) \leq 1$, we obtain $\mathcal{D}_{u} \leq 0$. Now, we deal with the case that $\operatorname{deg}_{\text {in }}(u) \geq 2$ and $\operatorname{deg}_{\text {out }}(u) \geq 2$. Since $\operatorname{deg}_{\leftrightarrow}(u) \leq \min \left(\operatorname{deg}_{\text {in }}(u), \operatorname{deg}_{\text {out }}(u)\right)$, a node that fulfils the bypassability criterion also fulfils $\operatorname{deg}_{\text {in }}(u) \cdot \operatorname{deg}_{\text {out }}(u) \leq c \cdot\left(\operatorname{deg}_{\text {in }}(u)+\operatorname{deg}_{\text {out }}(u)\right)+\min \left(\operatorname{deg}_{\text {in }}(u), \operatorname{deg}_{\text {out }}(u)\right)$. The inequality $x \cdot y \leq c(x+y)+\min (x, y)$ has only finitely many solutions $(x, y)$ for $x, y \in \mathbb{N}, x, y \geq 2$ if $c \in \mathbb{R}$ is a constant less than 2 . Consider the solution $(x, y)$ that maximises $k:=x \cdot y$. If there is no solution, take $k:=0$. Note that $k$ is a constant that only depends on the constant $c$. We can conclude that $\mathcal{D}_{u} \leq k$.

Each node from $V_{\ell}$ is bypassed at most once. For each bypassed node, the number of edges in the (tentative) core is increased by at most $k$. Therefore, $\left|E_{\ell}^{\prime}\right| \leq k \cdot\left|V_{\ell}\right|+\left|E_{\ell}\right|$.

If we used \#shortcuts $\leq \max \left(\operatorname{deg}_{\text {in }}(u), \operatorname{deg}_{\text {out }}(u)\right)$ as bypassability criterion, we would get a contraction that would be very similar to our earlier trees-and-lines method [23]. Note that the general version presented above allows a more effective contraction by setting $c$ appropriately.

Limiting Component Sizes. To reduce the observed maximum query time, we implement a limit on the number of hops a shortcut may represent. By this means, the sizes of the components of bypassed nodes are reduced-in particular, the first contraction step tended to create quite large components of bypassed nodes so that it took a long time to leave such a component when the search was started from within it.

\section{Query}

Our highway query algorithm is a modification of the bidirectional version of Dijkstra's algorithm. Note that in contrast to the construction, during the query we need not to keep track of ambiguous shortest paths. For now, we assume that the search is not aborted when both search scopes meet. This matter is dealt with in Section 5.3. We only describe the modifications of the forward search since forward and backward search are symmetric. In addition to the distance from the source, each node is associated with the search level and the gap to the 'next applicable neighbourhood border'. The search starts at the source node $s$ in level 0. First, a local search in the neighbourhood of $s$ is performed, i.e., the gap to the next border is set to the neighbourhood radius of $s$ in level 0 . When a node $v$ is settled, it adopts the gap of its parent $u$ minus the length of the edge $(u, v)$. As long as we stay inside the current neighbourhood, everything works as usual. However, if an edge $(u, v)$ crosses the neighbourhood border (i.e., the length of the edge is greater than the gap), we switch to a higher search level $\ell$. The node $u$ becomes an entrance point to the higher level. If the level of the edge $(u, v)$ is less than the new search level $\ell$, the edge is not relaxed-this is one of the two restrictions that cause the speedup in comparison to Dijkstra's algorithm (Restriction 1). Otherwise, the edge is relaxed: $v$ adopts the new search level $\ell$ and the gap to the border of the neighbourhood of $u$ in level $\ell$ since $u$ is the corresponding entrance point to level $\ell$. 
We have to deal with the special case that an entrance point to level $\ell$ does not belong to the core of level $\ell$. In this case, the search is continued inside a component of bypassed nodes till the level- $\ell$ core is entered, i.e., a node $u \in V_{\ell}^{\prime}$ is settled. At this point, $u$ is assigned the gap to the border of the level- $\ell$ neighbourhood of $u$. Note that before the core is entered (i.e., inside a component of bypassed nodes), the gap has been infinity (according to R1). To increase the speedup, we introduce another restriction (Restriction 2): when a node $u \in V_{\ell}^{\prime}$ is settled, all edges $(u, v)$ that lead to a bypassed node $v \in B_{\ell}$ in search level $\ell$ are not relaxed, i.e., once entered the core, we will never leave it again.

Figure 7 gives a detailed example of the forward search of a highway query. The search starts at node $s$. The gap of $s$ is initialised to the distance from $s$ to the border of the neighbourhood of $s$ in level 0 . Within the neighbourhood of $s$, the search process corresponds to a standard Dijkstra search. The edge that leads to $u$ leaves the neighbourhood. It is not relaxed due to Restriction 1 since the edge belongs only to level 0 . In contrast, the edge that leaves $s_{1}$ is relaxed since its level allows to switch to level 1 in the search process. $s_{1}$ and its direct successor are bypassed nodes in level 1. Their neighbourhoods are unbounded, i.e., their neighbourhood radii are infinity so that the gap is set to infinity as well. At $s_{1}^{\prime}$, we leave the component of bypassed nodes and enter the core of level 1 . Now, the search is continued in the core of level 1 within the neighbourhood of $s_{1}^{\prime}$. The gap is set appropriately. Note that the edge to $v$ is not relaxed due to Restriction 2 since $v$ is a bypassed node. Instead, the direct shortcut to $s_{2}$ is used. Here, we switch to level 2. In this case, we do not enter the next level through a component of bypassed nodes, but we get directly into the core. The search is continued in the core of level 2 within the neighbourhood of $s_{2}^{\prime}$. And so on.

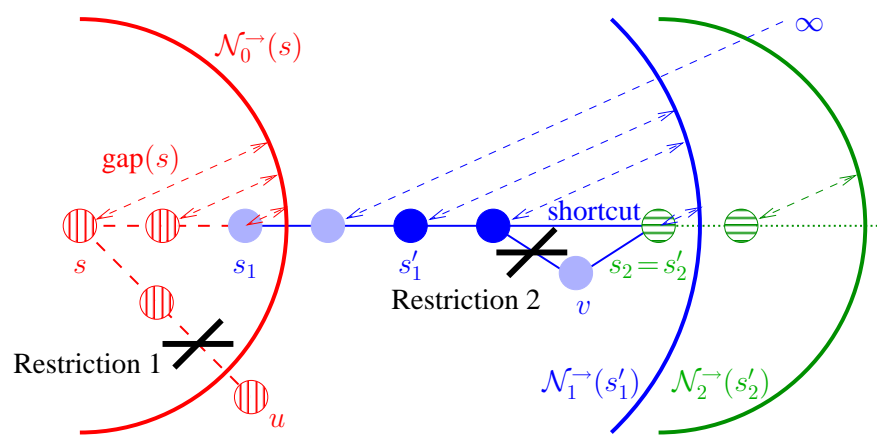

Fig. 7. A detailed example of a highway query. Only the forward search is depicted. Nodes in level 0,1 , and 2 are vertically striped, solid, and horizontally striped, respectively. In level 1, dark shades represent core nodes, light shades bypassed nodes. Edges in level 0, 1, and 2 are dashed, solid, and dotted, respectively.

Despite of Restriction 1, we always find the optimal path since the construction of the highway hierarchy guarantees that the levels of the edges that belong to the optimal path are sufficiently high so that these edges are not skipped. Restriction 2 does not invalidate the correctness of the algorithm since we have introduced shortcuts that bypass the nodes that do not belong to the core. Hence, we can use these shortcuts instead of the original paths.

\subsection{The Basic Algorithm}

We use two priority queues $\vec{Q}$ and $\overleftarrow{Q}$, one for the forward search and one for the backward search. For each search direction, a node $u$ is associated with a triple $(\delta(u), \ell(u), \operatorname{gap}(u))$, which we often call key. It consists of the (tentative) distance $\delta(u)$ from $s$ (or $t$ ) to $u$, the search level $\ell(u)$, and the gap $\operatorname{gap}(u)$ to the next applicable neighbourhood border. Only the 
first component $\delta(u)$ is used to decide the priority within the queue. ${ }^{2}$ We use the remaining two components for a tie breaking rule in the case that the same node is reached with two different keys $k:=\left(\delta, \ell\right.$, gap) and $k^{\prime}:=\left(\delta^{\prime}, \ell^{\prime}\right.$, gap $\left.^{\prime}\right)$ such that $\delta=\delta^{\prime}$. Then, we prefer $k$ to $k^{\prime}$ iff $\ell>\ell^{\prime}$ or $\ell=\ell^{\prime} \wedge$ gap $<$ gap $^{\prime}$. Note that any other tie breaking rule (or even omitting an explicit rule) will yield a correct algorithm. However, the chosen rule is most aggressive and has a positive effect on the performance. Figure 8 contains the pseudo-code of the highway query algorithm.

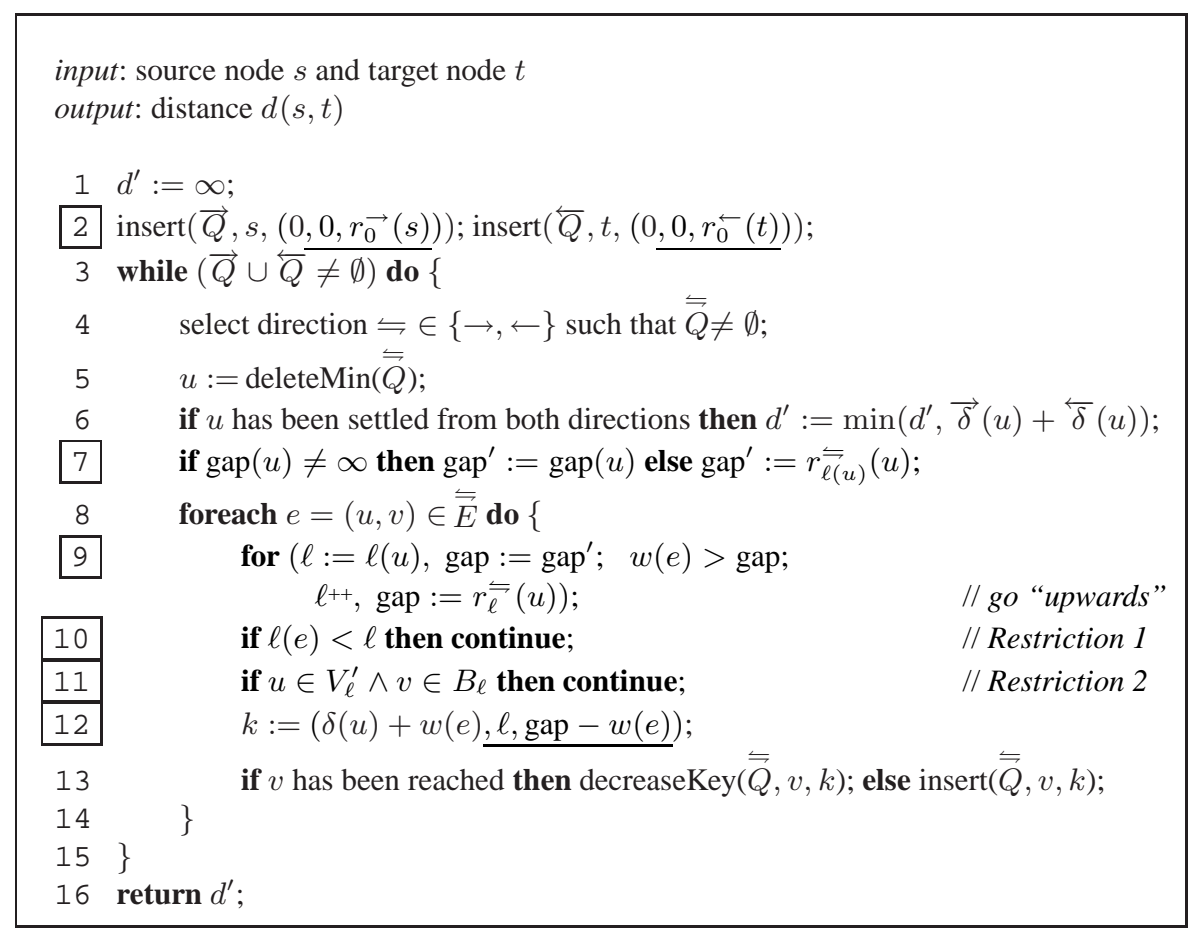

Fig. 8. The highway query algorithm. Differences to the bidirectional version of Dijkstra's algorithm are marked: additional and modified lines have a framed line number; in modified lines, the modifications are underlined.

Remarks:

- Line 4: The correctness of the algorithm does not depend on the strategy that determines the order in which the forward and the backward searches are processed. However, the choice of the strategy can affect the running time in the case that an abort-on-success criterion is applied (see Section 5.3).

- Line 7: This line deals with the special case that the entrance point did not belong to the core when the current search level $\ell$ was entered, i.e., the gap was set to infinity. In this

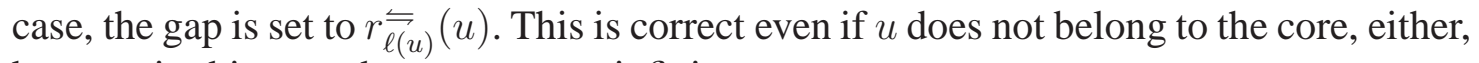
because in this case the gap stays at infinity.

- Line 9: It might be necessary to go upwards more than one level in a single step.

- Line 13: In the decreaseKey operation, the old key of $v$ is only replaced by $k$ if the above mentioned condition is fulfilled, i.e., if (a) the tentative distance is improved or (b) stays unchanged while the tie breaking rule succeeds. In the latter case (b), no priority queue operation is invoked since the priority (the tentative distance) has not changed. ${ }^{3}$

\footnotetext{
${ }^{2}$ If the search direction is not clear from the context, we will explicitly write $\vec{\delta}(u)$ and $\overleftarrow{\delta}(u)$ to distinguish between $u$ 's priority in $\vec{Q}$ and $\overleftarrow{Q}$

${ }^{3}$ That way, we also avoid problems that otherwise could arise when an already settled node is reached once again via a zero weight edge.
} 
Algorithmic Details. If we group the outgoing edges $(u, v)$ of each node $u$ by level, we can avoid looking at edges $(u, v)$ in levels $\ell(u, v)<\ell(u)$ since Restriction 1 would always apply to them. We can do without explicitly testing Restriction 2 if all edges $(u, v)$ with $k:=\ell(u, v), u \in V_{k}^{\prime}$, and $v \in B_{k}$ have been downgraded to level $k-1$. Then, the test of Restriction 1 also covers Restriction 2.

\subsection{Proof of Correctness}

Difficulties. Although the basic concepts (e.g. the definition of the highway network) and the algorithm are quite simple, the proof of correctness gets surprisingly complicated. The main reason for that is the fact that we cannot prove that the shortest path is found since there might be several shortest paths of the same length. We could assume that the shortest paths in the input are unique or that the uniqueness can be guaranteed by adding small fractions to the edge weights as it is done by other authors who face similar problems. However, the former would be too restrictive since usually, in real-world road networks, there are at least a few ambiguous instances, and a reliable realisation of the latter would be rather difficult. Furthermore, the introduction of shortcuts adds a lot of ambiguity even if it was not present in the input.

Therefore, if we pick any shortest path $P$ to show that it is found by the query algorithm, it can happen that a node $u$ on $P$ is settled from another node than its predecessor on $P$. Of course, in this case, $u$ will still be assigned the optimal distance from the source, but the search level and the distance to the next neighbourhood border may be different than expected so that we have to adapt to the new situation.

Outline. We face the above mentioned difficulties in the following way: First, we show that the algorithm terminates and deal with the special case that no path from the source to the target exists (Section A.1). Then, we introduce some definitions and concepts that will be useful in the main part of the correctness proof. In Section A.2, we define for a given path, a corresponding contracted path and an expanded path, where subpaths in the original graph are replaced by shortcuts or vice versa, respectively. In Section A.3, we first define the concepts of last neighbour and first core node, which, iteratively applied, lead to an unidirectional labelling of a given path. Figure 9 gives an example. Applying a forward and a backward labelling to the same path then allows the definition of a meeting level and a meeting point (Figure 10). The latter requires a case distinction since the forward and backward labelling may either meet in some core or in some component of bypassed nodes. Finally, we introduce the term highway path, a path whose properties exactly comply with the two restrictions of the query algorithm. Figure 11 gives an example.

In Section A.4, we deal with the reachability along a highway path. Basically, we show that if the query has settled some node $u$ on a highway path with the appropriate key, then

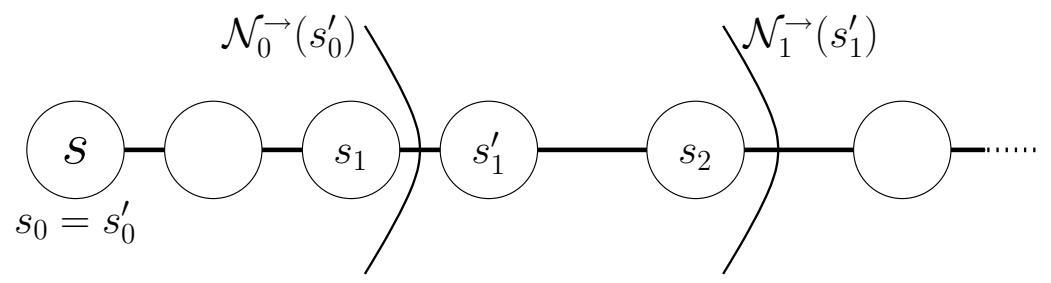

Fig. 9. Example for a forward labelling of a path $P$. The labels $s_{0}$ and $s_{0}^{\prime}$ are set to $s$ (base case). The node $s_{1}$ is the last neighbour of $s_{0}^{\prime}$ (denoted by $\vec{\omega}_{0}^{P}\left(s_{0}^{\prime}\right)$ ), the node $s_{1}^{\prime}$ is the first level-1 core node (denoted by $\vec{\alpha}_{1}^{P}\left(s_{1}\right)$ ), $s_{2}$ is the last neighbour of $s_{1}^{\prime}$, and so on. 


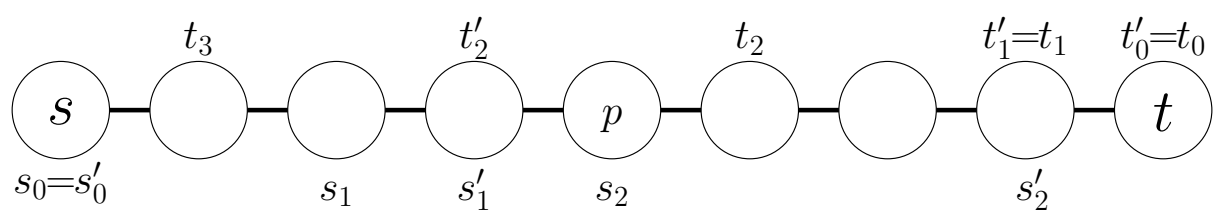

Fig. 10. Example for a forward and backward labelling (depicted below and above the nodes, respectively). The meeting level is 2 , the meeting point is $p$.

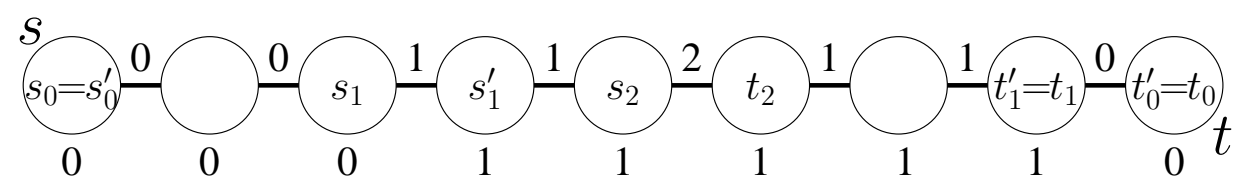

Fig. 11. Example for a highway path. Each edge belongs at least to the given level, each node at least to the given core level.

$u$ 's successor on that path can be reached from $u$ with the appropriate key as well (Lemmas 6 and 7, which are proved using the auxiliary Lemma 5). In other words, if there is a highway path, the query can follow the path (at least if there was no ambiguity).

In Section A.5, we use all concepts and lemmas introduced in the preceding sections to conduct the actual correctness proof, where we also deal with ambiguous paths. The general idea is to say that at any point the query algorithm has some valid state consisting of a shortest $s$ - $t$-path $P$ and two nodes $u \preceq \bar{u}$ that split $P$ into three parts such that the first and the third part are paths in the forward and backward search tree, respectively, and the second part is a contracted path. For such a valid state, we can prove that any node on the first and third part has been settled with the appropriate key (Lemma 8). Furthermore, we can show that $P$ is a highway path (Lemma 9 ).

When the algorithm is started, the nodes $s$ and $t$ are settled and some shortest $s$ - $t$-path $P$ in the original graph exists. (The special case that no $s$-t-path exists has already been dealt with.) Consequently, our initial state is composed of the contracted version of $P$ and the nodes $s$ and $t$, which makes it a valid state. A final state is a valid state where forward and backward search have met, i.e., they have settled a common node $u=\bar{u}$. Originally, we wanted to show that a shortest path is found. Now, we see (in Lemma 10) that it is sufficient to prove that a final state is reached.

We have already defined the meeting point $p$ on a path. We fall back on this definition and intend to prove that forward and backward search meet at $p$. When we look at any valid non-final state, it is obvious that at least one search direction can proceed to get closer to $p$, i.e., we have $u \prec p$ or $p \prec \bar{u}$ (Lemma 11). We pick such a non-blocked search direction. Let us assume w.l.o.g. that we picked the forward direction. We know that $u$ has been settled with the appropriate key and that $P$ is an optimal highway path (Lemmas 8 and 9). Due to the 'reachability along a highway path' (Lemmas 6 and 7), we can conclude that $u$ 's successor $v$ can be reached with the appropriate key as well, in particular with the optimal distance from $s$. A node that can be reached with the optimal distance will also be settled at some point with the optimal distance. However, we cannot be sure that $v$ is settled with $u$ as its parent since the shortest path from $s$ to $v$ might be ambiguous. At this point the state concept gets handy: we just replace the subpath of $P$ from $s$ to $v$ with the path in the search tree that actually has been taken yielding a path $P^{+}$; we obtain a new state that consists of $P^{+}$and the nodes $v$ and $\bar{u}$. We prove that the new state is valid (Lemma 12).

Thus, we can show that from any valid non-final state another valid state is reached at some point. We also show in Lemma 12 that we cannot get into some cycle of states since 
in each step the length of the middle part of the path is decreased. Hence, starting from the initial state, eventually a final state is reached so that a shortest path is found (Theorem 5).

The actual proof can be found in Appendix A.

\subsection{Optimisations}

Rearranging Nodes. Similar to [20], after the construction has been completed, we rearrange the nodes by core level, which improves locality for the search in higher levels and, thus, reduces the number of cache misses.

Speeding Up the Search in the Topmost Level. Let us assume that we have a distance table that contains for any node pair $s, t \in V_{L}^{\prime}$ the optimal distance $d_{L}(s, t)$. Such a table can be precomputed during the preprocessing phase by $\left|V_{L}^{\prime}\right|$ SSSP searches in $G_{L}^{\prime}$. Using the distance table, we do not have to search in level $L$. Instead, when we arrive at a node $u \in V_{L}^{\prime}$ that leads to level $L$, we add $u$ to the initially empty set $\vec{I}$ or $\overleftarrow{I}$ depending on the search direction; we do not relax the edge that leads to level $L$. After all entrance points have been encountered, we consider all pairs $(u, v) \in \vec{I} \times \overleftarrow{I}$ and compute the minimum path length $D:=\vec{\delta}(u)+d_{L}(u, v)+\overleftarrow{\delta}(v)$. Then, the length of the shortest $s$-t-path is the minimum of $D$ and the length $d^{\prime}$ of the tentative shortest path found so far (in case that the search scopes have already met in a level $<L$ ).

For the sake of a simple incorporation of this optimisation into the highway query algorithm, we slightly revise the properties R1 and R2: we use two distinguishable values $\infty_{1}$ and $\infty_{2}$ that are larger than any real number and set $r_{\ell} \leftrightharpoons(u):=\infty_{1}$ for any $\ell$ and any node

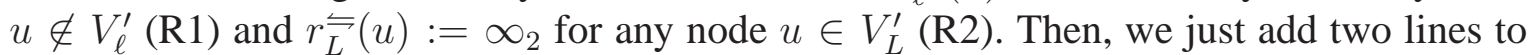
Figure 8 and modify Line 16 :

between Lines 7 and 8:

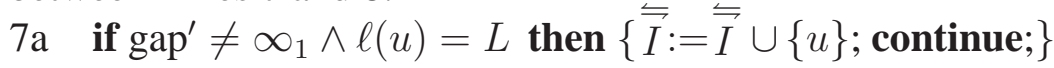

between Lines 11 and 12:

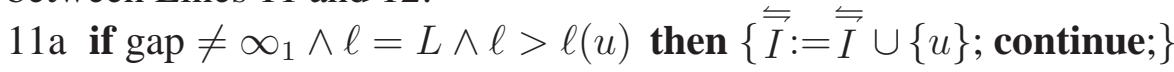

16 return $\min \left(\left\{d^{\prime}\right\} \cup\left\{\vec{\delta}(u)+d_{L}(u, v)+\overleftarrow{\delta}(v) \mid u \in \vec{I}, v \in \overleftarrow{I}\right\}\right)$

In Section A.6, we show that our proof of correctness still holds when the distance table optimisation is applied.

Abort on Success. In the bidirectional version of Dijkstra's algorithm, we can abort the search as soon as both search scopes meet. Unfortunately, this would be incorrect for our highway query algorithm. Therefore, we use a more conservative criterion: after a tentative shortest path $P^{\prime}$ has been encountered (i.e., after both search scopes have met), the forward (backward) search is not continued if the minimum element $u$ of the forward (backward) queue has a key $\delta(u) \geq w\left(P^{\prime}\right)$. Obviously, the correctness of the algorithm is not invalidated by this abort criterion. In [23] we tried using more sophisticated criteria in order to reduce the search space. However, it turned out that this simple criterion, since it can be evaluated so efficiently, yields better query times in spite of a somewhat larger search space. Note that when the distance table optimisation is used and random queries are performed, our simple abort criterion is very close to an optimal criterion even with respect to the search space size: our experiments indicate that less than $1 \%$ of the search space is visited after the first meeting of forward and backward search. 


\subsection{Outputting Complete Path Descriptions}

The highway query algorithm in Figure 8 only computes the distance from $s$ to $t$. In order to determine the actual shortest path, we need to store pointers from each node to its parent in the search tree. Note that the algorithm could be easily modified to compute all shortest paths between $s$ and $t$ by just storing more than one parent pointer in case of ambiguities. However, subsequently, we only deal with a single shortest path.

We face two problems in order to determine a complete description of the shortest path: (a) we have to bridge the gap between the forward and backward topmost core entrance points (in case that the distance table optimisation is used) and (b) we have to expand the used shortcuts to obtain the corresponding subpaths in the original graph.

Problem (a) can be solved using a simple algorithm: We start with the forward core entrance point $u$. As long as the backward entrance point $v$ has not been reached, we consider all outgoing edges $(u, w)$ in the topmost core and check whether $d_{L}(u, w)+d_{L}(w, v)=$ $d_{L}(u, v)$; we pick an edge $(u, w)$ that fulfils the equation, and we set $u:=w$. The check can be performed using the distance table. It allows us to greedily determine the next hop that leads to the backward entrance point.

Problem (b) can be solved without using any extra data (Variant 1). For each shortcut $(u, v) \in S_{\ell}$ on the shortest path, we perform a search from $u$ to $v$ in order to determine the represented path in $G_{\ell}$. This search can be accelerated by using the knowledge that the first edge of the path enters a component $C$ of bypassed nodes, the last edge leads to $v$, and all other edges are situated within the component $C$. The represented path in $G_{\ell}$ may contain shortcuts from sets $S_{k}, k<\ell$, which are expanded recursively. In the end, we obtain the represented path from $u$ to $v$ in the original graph.

However, if a fast output routine is required, it is necessary to spend some additional space to accelerate the unpacking process. We use a rather sophisticated data structure to represent unpacking information for the shortcuts in a space-efficient way (Variant 2). In particular, we do not store a sequence of node IDs that describe a path that corresponds to a shortcut, but we store only hop indices: for each edge $(u, v)$ on the path that should be represented, we store its rank within the ordered group of edges that leave $u$. Since in most cases the degree of a node is very small, these hop indices can be stored using only a few bits. The unpacked shortcuts are stored in a recursive way, e.g., the description of a level-2 shortcut may contain several level-1 shortcuts. Accordingly, the unpacking procedure works recursively.

To obtain a further speed-up, we have a variant of the unpacking data structures (Variant 3) that caches the complete descriptions-without recursions-of all shortcuts that belong to the topmost level, i.e., for these important shortcuts that are frequently used, we do not have to use a recursive unpacking procedure, but we can just append the corresponding subpath to the resulting path.

\subsection{Turning Restrictions}

A turning restriction (in its simplest and most common form) is expressed as an edge pair $((u, v),(v, w))$ : the edge $(v, w)$ must not be traversed if the node $v$ has been reached via the edge $(u, v)$. Dealing with turning restrictions is a well-studied problem [33,34]. In principle, there are two basic approaches: modifying the query algorithm or modelling the restrictions into the graph, which introduces additional artificial nodes and edges at affected road junctions. The latter technique can be applied irrespective of the used query algorithm.

In case of highway hierarchies, we expect that modelling turning restrictions into the graph only slightly deteriorates the performance since the artificial nodes usually have a 
very small degree so that most of them get bypassed in the very first contraction step. Furthermore, turning restrictions are often encountered at local streets that are not promoted to high levels of the hierarchy so that the negative impact is bounded to the lower levels. With respect to memory consumption, it is important to note that after the preprocessing has been completed, artificial nodes and edges at road junctions that only belong to level 0 can be abandoned provided that the query algorithm (which in level 0 just corresponds to Dijkstra's algorithm) is modified appropriately to handle turning restrictions.

\section{Experiments}

Apart from Section 6.8, all experimental results refer to the scenario where we only want to compute the shortest-path length between two nodes without outputting the actual route. Turning restrictions are exclusively handled in Section 6.9.

\subsection{Implementation}

We implemented highway hierarchies in $\mathrm{C}++$, using the $\mathrm{C}++$ Standard Template Library and making extensive use of generic programming techniques using $\mathrm{C}++$ 's template class mechanism. As graph data structure, we use our own implementation of an adjacency array extended by an additional layer that contains level-specific data for each node and level that the node belongs to. We use 32 bits to store edge weights and path lengths. Binary heaps are used as priority queues. Note that in case of road networks only a comparatively small number of decreaseKey-operations is performed. Furthermore, the number of nodes that are in the priority queue at the same time is very small in case of highway hierarchies (usually less than 100 nodes). Therefore, using a more sophisticated priority queue implementation is not likely to increase the performance significantly. For some more details on the implementation, we refer to Appendix B.

\subsection{Environment and Instances}

The experiments were done on one core of a single AMD Opteron Processor 270 clocked at 2.0 GHz with $8 \mathrm{~GB}$ main memory and $2 \times 1 \mathrm{MB}$ L2 cache, running SuSE Linux 10.0 (kernel 2.6.13). The program was compiled by the GNU C++ compiler 4.0.2 using optimisation level 3 .

We deal with the road networks of Western Europe ${ }^{4}$ and of the USA (without Hawaii) and Canada. Both networks have been made available for scientific use by the company PTV AG. The original graphs contain for each edge a length and a road category, e.g., motorway, national road, regional road, urban street. We assign average speeds to the road categories $^{5}$, compute for each edge the average travel time, and use it as weight. In addition, we perform experiments on a publicly available version of the US road network (without Alaska and Hawaii) that was obtained from the TIGER/Line Files [35]. However, in contrast to the PTV data, the TIGER graph is undirected, planarised and distinguishes only between four road categories $(40,60,80,100 \mathrm{~km} / \mathrm{h})$, in fact $91 \%$ of all roads belong to the slowest category so that you cannot discriminate them.

Table 1 summarises important properties of the used road networks and the key results of the experiments.

\footnotetext{
${ }^{4}$ Austria, Belgium, Denmark, France, Germany, Italy, Luxembourg, the Netherlands, Norway, Portugal, Spain, Sweden, Switzerland, and the UK

${ }^{5}$ For Europe: $10,20, \ldots, 130 \mathrm{~km} / \mathrm{h}$; for USA/CAN: 16, 24, 32, 40, 56, 64, 72, 80, 88, 96, 96, 104, $112 \mathrm{~km} / \mathrm{h}$.
} 
Table 1. Overview of the used road networks and key results. 'Øoverhead/node' accounts for the additional memory that is needed by our highway hierarchy approach (divided by the number of nodes) compared to a space-efficient bidirectional implementation of Dijkstra's algorithm. Query times are average values based on 10000 random $s$-t-queries. 'Speedup' refers to a comparison with Dijkstra's algorithm (unidirectional). Worst case is an upper bound for any possible query in the respective graph.

\begin{tabular}{llrrr}
\hline & & Europe & USA/CAN & USA (Tiger) \\
\hline \multirow{3}{*}{ INPUT } & \#nodes & 18029721 & 18741705 & 24278285 \\
& \#directed edges & 42199587 & 47244849 & 58213192 \\
& \#road categories & 13 & 13 & 4 \\
\hline \multirow{2}{*}{ PARAM. } & average speeds [km/h] & $10-130$ & $16-112$ & $40-100$ \\
& $H$ & 30 & 40 & 40 \\
\hline \multirow{2}{*}{ PREPROC. } & CPU time [min] & 13 & 17 & 15 \\
& $\varnothing$ overhead/node [byte] & 48 & 46 & 34 \\
\hline \multirow{6}{*}{ QUERY } & CPU time [ms] & 0.61 & 0.83 & 0.67 \\
& \#settled nodes & 709 & 871 & 925 \\
& \#relaxed edges & 2531 & 3376 & 3823 \\
& speedup (CPU time) & 9935 & 7259 & 9303 \\
& speedup (\#settled nodes) & 12715 & 10750 & 12889 \\
& worst case (\#settled nodes) & 2388 & 2428 & 2505 \\
\hline
\end{tabular}

\subsection{Parameters}

Default Settings. Unless otherwise stated, the following default settings apply. We use the maverick factor $f=2(i-1)$ for the $i$-th iteration of the construction procedure, the contraction rate $c=2$, the shortcut hops limit 10, and the neighbourhood sizes $H$ as stated in Table 1-the same neighbourhood size is used for all levels of a hierarchy. First, we contract the original graph. ${ }^{6}$ Then, we perform five iterations of our construction procedure, which determines a highway network and its core. Finally, we compute the distance table between all level-5 core nodes.

Self-Similarity. For two levels $\ell$ and $\ell+1$ of a highway hierarchy, the shrinking factor is the ratio between $\left|E_{\ell}^{\prime}\right|$ and $\left|E_{\ell+1}^{\prime}\right|$. In our experiments, we observed that the highway hierarchies of Europe and the USA were almost self-similar in the sense that the shrinking factor remained nearly unchanged from level to level when we used the same neighbourhood size $H$ for all levels-provided that $H$ was not too small.

Figure 12 demonstrates the shrinking process for Europe. Note that the first contraction step is not shown. In contrast to our default settings, we do not stop after five iterations. For most levels and $H \geq 70$, we observe an almost constant shrinking factor (which appears as a straight line due to the logarithmic scale of the y-axis). The greater the neighbourhood size, the greater the shrinking factor. The last iteration is an exception: the highway network collapses, i.e., it shrinks very fast because nodes that are close to the border of the network usually do not belong to the next level of the highway hierarchy, and when the network gets small, almost all nodes are close to the border. In case of the smallest neighbourhood size $(H=30)$, the shrinking factor gets so small that the network does not collapse even after 14 levels have been constructed.

Varying the Neighbourhood Size. Note that in order to simplify the experimental setup all results in the remainder of Section 6.3 have been obtained without rearranging nodes by level. However, since we want to demonstrate the effects of choosing different parameter settings, the relative performance is already very meaningful.

\footnotetext{
${ }^{6}$ In Section 3, we gave the definition of the highway hierarchies where we first construct a highway network and then contract it. We decided to change this order in the experiments, i.e., to start with an initial contraction phase, since we observed a better performance in this case.
} 


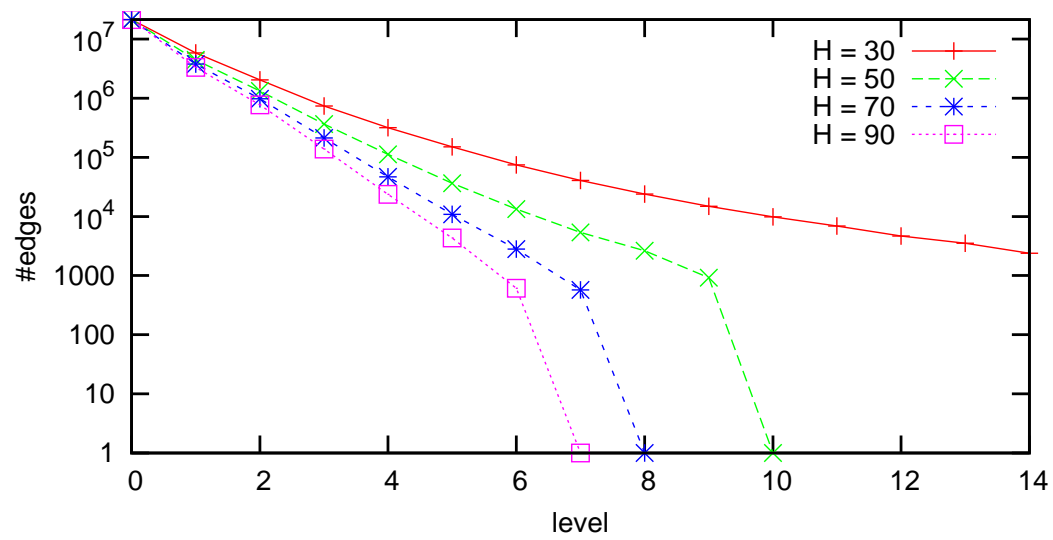

Fig. 12. Shrinking of the highway networks of Europe. For different neighbourhood sizes $H$ and for each level $\ell$, we plot $\left|E_{\ell}^{\prime}\right|$, i.e., the number of edges that belong to the core of level $\ell$.

In one test series (Figure 13), we used all the default settings except for the neighbourhood size $H$, which we varied in steps of 5. On the one hand, if $H$ is too small, the shrinking of the highway networks is less effective so that the level-5 core is still quite big. Hence, we need much time and space to precompute and store the distance table. On the other hand, if $H$ gets bigger, the time needed to preprocess the lower levels increases because the area covered by the local searches depends on the neighbourhood size. Furthermore, during a query, it takes longer to leave the lower levels in order to get to the topmost level where the distance table can be used. Thus, the query time increases as well. We observe that the preprocessing time is minimised for neighbourhood sizes around 40. In particular, the optimal neighbourhood size does not vary very much from graph to graph. In other words, if we used the same parameter $H$, say 40, for all road networks, the resulting performance would be very close to the optimum. Obviously, choosing different neighbourhood sizes leads to different space-time trade-offs.
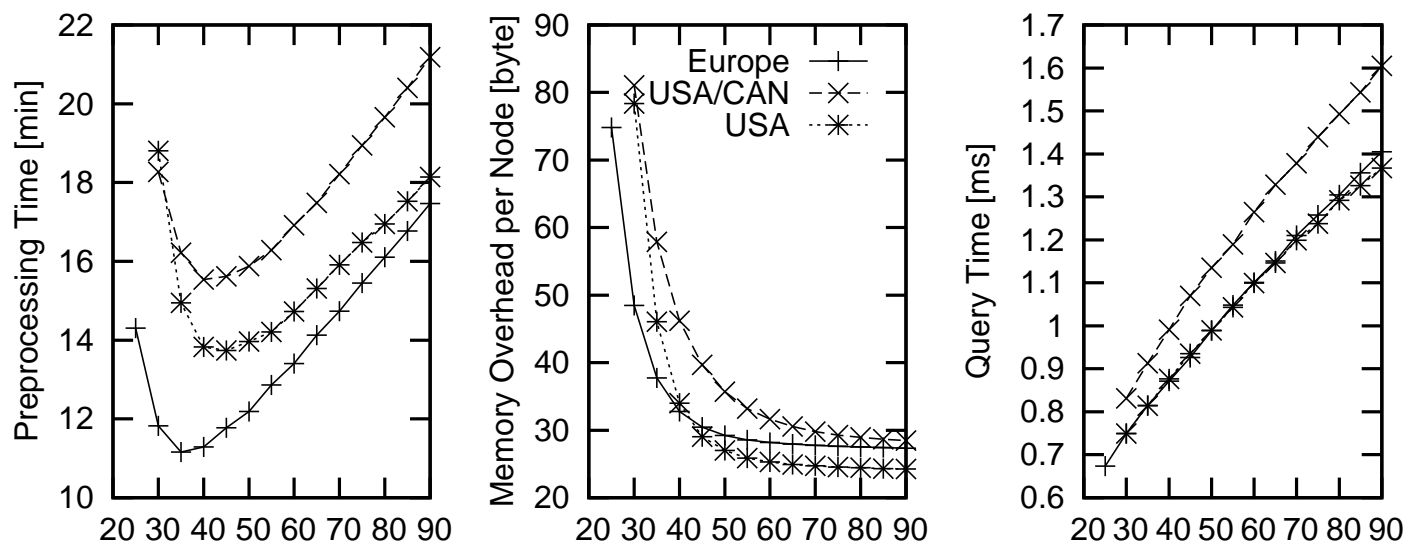

Fig. 13. Preprocessing and query performance depending on the neighbourhood size $H$.

Varying the Contraction Rate. In another test series (Table 2a), we did not use a distance table, but repeated the construction process until the topmost level was empty or the hierarchy consisted of 15 levels. We varied the contraction rate $c$ from 0.5 to 2.5. In case of $c=0.5$ (and $H=30$ ), the shrinking of the highway networks does not work properly so that the topmost level is still very big. This yields huge query times. Choosing larger contraction rates 
reduces the preprocessing and query times since the cores and search spaces get smaller. However, the memory usage and the average degree are slightly increased since more shortcuts are introduced. Adding too many shortcuts $(c=2.5)$ further reduces the search space, but the number of relaxed edges increases so that the query times get worse.

Varying the Number of Levels. In a third test series (Table 2b), we used the default settings except for the number of levels, which we varied from 6 to 11. Note that the original graph and its core (i.e., the result of the first contraction step) counts as one level so that for example ' 6 levels' means that only five levels are constructed. In each test case, a distance table was used in the topmost level. The construction of the higher levels of the hierarchy is very fast and has no significant effect on the preprocessing times. In contrast, using only six levels yields a rather large distance table, which somewhat slows down the preprocessing and increases the memory usage. However, in terms of query times, ' 6 levels' is the optimal choice since using the distance table is faster than continuing the search in higher levels. We omitted experiments with less levels since this would yield very large distance tables consuming very much memory.

Results for further combinations of neighbourhood size, contraction rate, and number of levels can be found in Table 5 and 6 in Appendix C.

Table 2. Preprocessing and query performance for the European road network depending on the contraction rate $c$ (a) and the number of levels (b). 'overhead' denotes the average memory overhead per node in bytes.

\begin{tabular}{|c|c|c|c|c|c|c|}
\hline $\begin{array}{l}\text { contr. } \\
\text { rate } c\end{array}$ & \multicolumn{3}{|c|}{$\begin{array}{l}\text { PREPROCESSING } \\
\text { time over- } \varnothing \mathrm{deg} . \\
\text { [min] head }\end{array}$} & $\begin{array}{l}\text { time } \\
{[\mathrm{ms}]}\end{array}$ & $\begin{array}{r}\text { QUERY } \\
\text { \#settled } \\
\text { nodes }\end{array}$ & $\begin{array}{r}\text { \#relaxed } \\
\text { edges }\end{array}$ \\
\hline 0.5 & 83 & 30 & 3.2 & 391.73 & 472326 & 1023944 \\
\hline 1.0 & 15 & 28 & 3.7 & 5.48 & 6396 & 23612 \\
\hline 1.5 & 11 & 28 & 3.8 & 1.93 & 1830 & 9281 \\
\hline 2.0 & 11 & 29 & 4.0 & 1.85 & 1542 & 8913 \\
\hline 2.5 & 11 & 30 & 4.1 & 1.96 & 1489 & 9175 \\
\hline
\end{tabular}

(a)

\begin{tabular}{c|rr|rr}
\multirow{2}{*}{$\begin{array}{c}\text { \# } \\
\text { levels }\end{array}$} & $\begin{array}{r}\text { PREPROC. } \\
\text { time over- }\end{array}$ & \multicolumn{2}{|c}{ QUERe \#settled } \\
& timin & head & {$[\mathrm{ms}]$} & nodes \\
\hline 6 & 12 & 48 & 0.75 & 709 \\
7 & 10 & 34 & 0.93 & 852 \\
8 & 10 & 30 & 1.14 & 991 \\
9 & 10 & 30 & 1.35 & 1123 \\
10 & 10 & 29 & 1.54 & 1241 \\
11 & 10 & 29 & 1.67 & 1326 \\
\hline
\end{tabular}

(b)

\subsection{Local Queries}

For use in applications it is unrealistic to assume a uniform distribution of queries in large graphs such as Europe or the USA. On the other hand, it would be hardly more realistic to arbitrarily cut the graph into smaller pieces. Therefore, we decided to measure local queries within the big graphs: For each power of two $r=2^{k}$, we choose random sample points $s$ and then use Dijkstra's algorithm to find the node $t$ with Dijkstra $\operatorname{rank} \mathrm{rk}_{s}(t)=r$. We then use our algorithm to make an $s$-t-query. By plotting the resulting statistics for each value $r=2^{k}$, we can see how the performance scales with a natural measure of difficulty of the query. Figure 14 shows the query times. Note that for ranks up to $2^{18}$ the median query times are scaling quite smoothly and the growth is much slower than the exponential increase we would expect in a plot with logarithmic $x$ axis, linear $y$ axis, and any growth rate of the form $r^{\rho}$ for Dijkstra rank $r$ and some constant power $\rho$; the curve is also not the straight line one would expect from a query time logarithmic in $r$. For ranks $r \geq 2^{19}$, the query times hardly rise due to the fact that the all-pairs distance table can bridge the gap between the forward and backward search of these queries irrespective of dealing with a small or a large gap. In case of Europe and USA/CAN, the query times drop for $r=2^{24}$ since $r$ is only slightly smaller than the number of nodes so that the target lies close to the border of the respective road network which implies some kind of trivial sense of goal direction for the backward search (because, in the beginning, we practically cannot go into the wrong direction). 


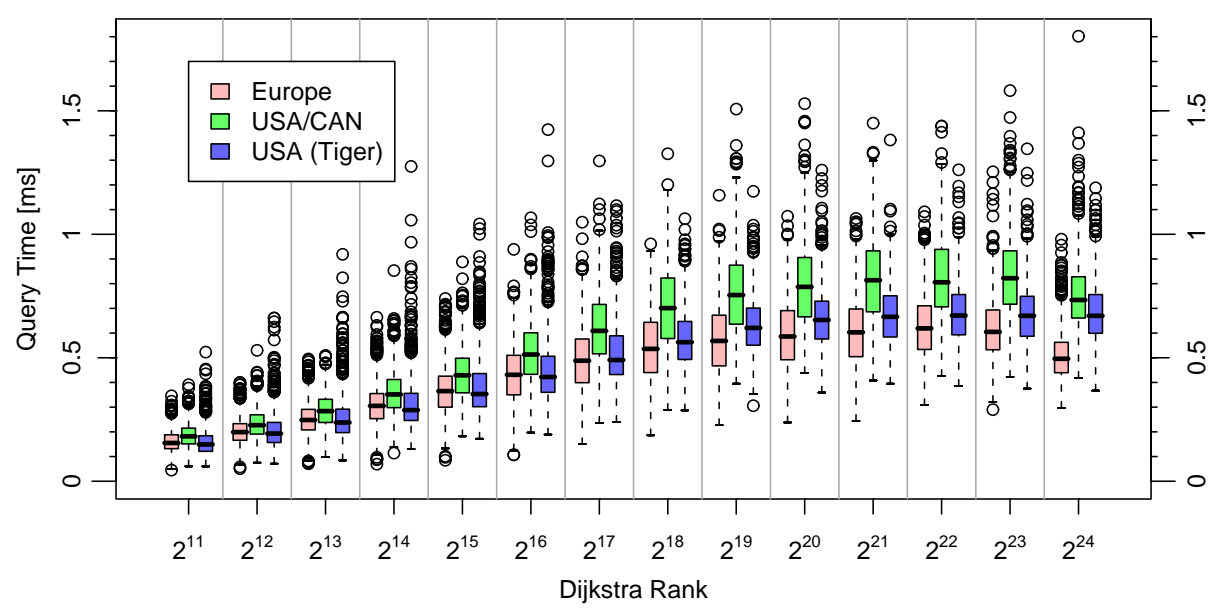

Fig. 14. Local queries. The distributions are represented as box-and-whisker plots [36]: each box spreads from the lower to the upper quartile and contains the median, the whiskers extend to the minimum and maximum value omitting outliers, which are plotted individually.

\subsection{Space Saving}

If we omit the first contraction step and use a smaller contraction rate ( $\Rightarrow$ less shortcuts), use a bigger neighbourhood size ( $\Rightarrow$ higher levels get smaller), and construct more levels before the distance table is used ( $\Rightarrow$ smaller distance table), the memory usage can be reduced considerably. In case of Europe, using seven levels, $H=100$, and $c=1$ yields an average overhead per node of 17 bytes. The construction and query times increase to $55 \mathrm{~min}$ and $1.10 \mathrm{~ms}$, respectively.

\subsection{Worst Case Upper Bounds}

By executing a query from each node of a given graph to an added isolated dummy node and a query from the dummy node to each actual node in the backward graph, we obtain a distribution of the search space sizes of the forward and backward search, respectively. We can combine both distributions to get an upper bound for the distribution of the search space sizes of bidirectional queries: when $\mathcal{F}_{\rightarrow}(x)\left(\mathcal{F}_{\leftarrow}(x)\right)$ denotes the number of source (target) nodes whose search space consists of $x$ nodes in a forward (backward) search, we define $\mathcal{F}_{\leftrightarrow}(z):=\sum_{x+y=z} \mathcal{F}_{\rightarrow}(x) \cdot \mathcal{F}_{\leftarrow}(y)$, i.e., $\mathcal{F}_{\leftrightarrow}(z)$ is the number of $s$ - $t$-pairs such that the upper bound of the search space size of a query from $s$ to $t$ is $z$. In particular, we obtain the upper bound $\max \left\{z \mid \mathcal{F}_{\leftrightarrow}(z)>0\right\}$ for the worst case without performing all $n^{2}$ possible queries.

Figure 15 visualises the distribution $\mathcal{F}_{\leftrightarrow}(z)$ as a histogram. In a similar way, we obtained a distribution of the number of entries in the distance table that have to be accessed during an $s$-t-query. While the average values are reasonably small (4066 in case of Europe), the worst case can get quite large (62 379). For example, accessing 62379 entries in a table of size $9351 \times 9351$ takes about $1.1 \mathrm{~ms}$, where 9351 is the number of nodes of the level-5 core of the European highway hierarchy. Hence, in some cases the time needed to determine the optimal entry in the distance table might dominate the query time. We could try to improve the worst case by introducing a case distinction that checks whether the number of entries that have to be considered exceeds a certain threshold. If so, we would not use the distance table, but continue with the normal search process. However, this measures would have only little effect on the average performance. 


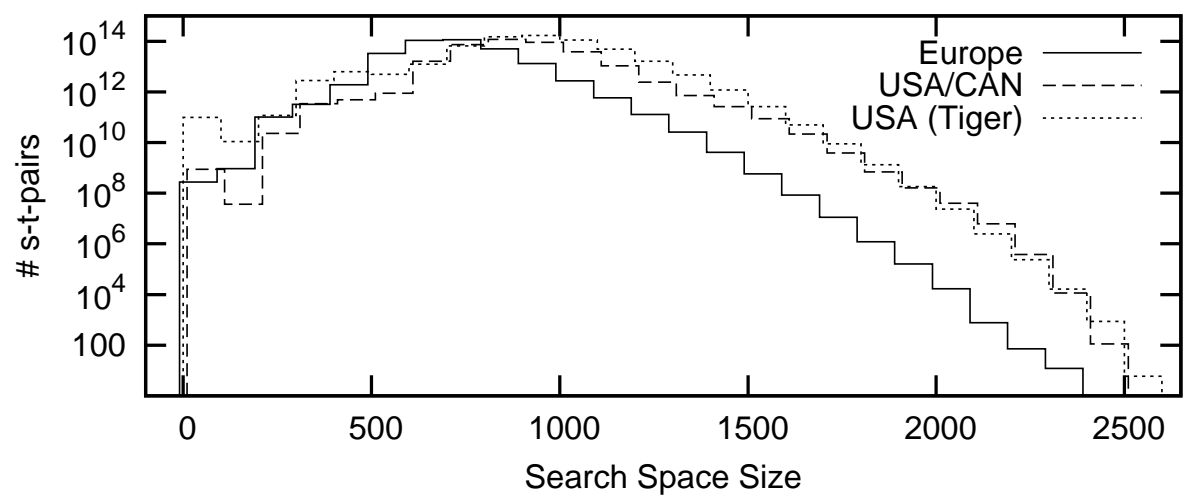

Fig. 15. Histogram of upper bounds for the search space sizes of $s$-t-queries. To increase readability, only the outline of the histogram is plotted instead of the complete boxes.

\subsection{Comparisons}

In Table 3, we compare our highway hierarchies with some of the most competitive methods where experimental results are available for the Western European and the US road network, namely with the REAL algorithm [20], transit-node routing [27], and highway-node routing [29]. For REAL and transit-node routing slightly smaller graphs were used, namely the largest connected component of each road network consisting of about $99 \%$ of all nodes. Experiments with the REAL algorithm have been performed on a slightly different machine (dual-processor, 2.4 GHz AMD Opteron).

Although a comparison is difficult since all approaches allow different choices of parameter settings yielding different space-time trade-offs, we can make some general statements: The strength of transit-node routing is clearly the extremely good query performance. Highway-node routing has an outstandingly low memory consumption, while the query times are competitive to highway hierarchies and REAL or even slightly superior in case of the US road network. Highway hierarchies can achieve very low preprocessing times or a quite low memory consumption, while query times are reasonably good in all cases. REAL's performance is similar to highway hierarchies except for the preprocessing times, which tend to be considerably higher.

It is very important to note that both highway-node routing and the implementation of transit-node routing considered in this section are based on our highway hierarchies. Thus, at the moment neither of these methods can supersede the highway hierarchies approach. Actually, the results for highway-node routing are better than the ones published in [29]

Table 3. Comparison between highway hierarchies (HH), the REAL algorithm, transit-node routing (TNR) and highwaynode routing (HNR). For the former three approaches, different parameter settings are examined. 'disk space' denotes the total amount of memory needed to store the preprocessed data including the original graph on disk.

\begin{tabular}{|c|c|c|c|c|c|c|c|c|}
\hline \multirow[b]{3}{*}{ method } & \multicolumn{4}{|c|}{ Europe } & \multicolumn{4}{|c|}{ USA (Tiger) } \\
\hline & PREPRC & CESSING & QUI & ERY & PREPR & OCESSING & QUI & ERY \\
\hline & $\begin{array}{l}\text { time } \\
{[\mathrm{min}]}\end{array}$ & $\begin{array}{c}\text { isk space } \\
{[\mathrm{MB}]}\end{array}$ & $\begin{array}{l}\text { time } \\
{[\mathrm{ms}]}\end{array}$ & $\begin{array}{c}\text { \#settled } \\
\text { nodes }\end{array}$ & $\begin{array}{l}\text { time } \\
{[\mathrm{min}]}\end{array}$ & $\begin{array}{c}\text { disk space } \\
{[\mathrm{MB}]}\end{array}$ & $\begin{array}{l}\text { time } \\
{[\mathrm{ms}]}\end{array}$ & $\begin{array}{c}\text { \#settled } \\
\text { nodes }\end{array}$ \\
\hline$\overline{\mathrm{HH}}$ & 13 & 1241 & 0.61 & 709 & 15 & 1324 & 0.67 & 925 \\
\hline $\mathrm{HH}$ (mem) & 55 & 697 & 1.10 & 1863 & 70 & 942 & 1.21 & 2143 \\
\hline REAL $(16,1)$ & 97 & 1849 & 1.22 & 814 & 64 & 3028 & 1.14 & 675 \\
\hline REAL $(64,16)$ & 141 & 1015 & 1.11 & 679 & 121 & 1575 & 1.05 & 540 \\
\hline TNR (eco) & 46 & 2304 & 0.0134 & N/A & 59 & 3073 & 0.0115 & N/A \\
\hline TNR (gen) & 164 & 4714 & 0.0056 & N/A & 205 & 6108 & 0.0049 & N/A \\
\hline HNR & 15 & 503 & 0.88 & 1017 & 16 & 640 & 0.50 & 760 \\
\hline
\end{tabular}


since in the meantime the highway hierarchies have improved and highway-node routing directly benefits from that.

\subsection{Outputting Complete Path Descriptions}

So far, we have reported only the times needed to compute the shortest-path length between two nodes. Now, we determine a complete description of the shortest path. In Table 4 we give the additional preprocessing time and the additional disk space for the unpacking data structures. Furthermore, we report the additional time that is needed to determine a complete description of the shortest path and to traverse ${ }^{7}$ it summing up the weights of all edges as a sanity check-assuming that the query to determine the shortest-path length has already been performed. That means that the total average time to determine a shortest path is the time given in Table 4 plus the query time given in previous tables ${ }^{8}$. Note that Variant 1 is no longer supported by the current version of our implementation so that the numbers in the first data row of Table 4 have been obtained with an older version and different settings.

We can conclude that even Variant 3 requires little additional preprocessing time and only a moderate amount of space. With Variant 3, the time for outputting the path remains considerably smaller than the time to determine the path length and a factor 3-5 smaller than using Variant 2. The US graph profits more than the European graph since it has paths with considerably larger hop counts, perhaps due to a larger number of degree two nodes in the input. Note that due to cache effects, the time for outputting the path using preprocessed shortcuts is likely to be considerably smaller than the time for traversing the shortest path in the original graph.

Table 4. Additional preprocessing time, additional disk space and query time that is needed to determine a complete description of the shortest path and to traverse it summing up the weights of all edges-assuming that the query to determine its lengths has already been performed. Moreover, the average number of hops-i.e., the average path length in terms of number of nodes-is given. The three algorithmic variants are described in Section 5.4.

\begin{tabular}{l|rrrr|rrrr} 
& \multicolumn{4}{|c|}{ Europe } & \multicolumn{4}{|c}{ USA (Tiger) } \\
& $\begin{array}{r}\text { preproc. } \\
\text { space }\end{array}$ & query & \# hops \\
& {$[\mathrm{s}]$} & {$[\mathrm{MB}]$} & {$[\mathrm{ms}]$} & (avg.) & preproc. & space & query & \# hops \\
{$[\mathrm{MB}]$} & {$[\mathrm{ms}]$} & (avg.) \\
\hline Variant 1 & 0 & 0 & 17.22 & 1366 & 0 & 0 & 39.69 & 4410 \\
Variant 2 & 69 & 126 & 0.49 & 1366 & 68 & 127 & 1.16 & 4410 \\
Variant 3 & 74 & 225 & 0.19 & 1366 & 70 & 190 & 0.25 & 4410
\end{tabular}

\subsection{Turning Restrictions}

We did an experiment with the German road network (a subgraph of our European network) and real-world turning restrictions (also provided by PTV) to verify our expectation that incorporating the restrictions into the graph has only a little effect on the performance. The results are positive: the preprocessing time does not change, the total number of nodes and edges in the highway hierarchy only increases by $4 \%$, and the query times rise by $3 \%$.

\subsection{Distance Metric}

When we apply a distance metric instead of the usual (and for most practical applications more relevant) travel time metric, the hierarchy that is inherent in the road network is less

\footnotetext{
${ }^{7}$ Note that we do not traverse the path in the original graph, but we directly scan the assembled description of the path.

${ }^{8}$ Note that in the current implementation outputting path descriptions and the feature to rearrange nodes by level are mutually exclusive. However, this is not a limitation in principle.
} 
distinct since the difference between fast and slow roads fades. We no longer observe the self-similarity in the sense that a fixed neighbourhood size yields an almost constant shrinking factor. Instead, we have to use an increasing sequence of neighbourhood sizes to ensure a proper shrinking. For Europe, we use $H=100,200,300,400,500$ to construct five levels before an all-pairs distance table is built. Constructing the hierarchy takes 34 minutes and entails a memory overhead of 36 bytes per node. On average, a random query then takes $4.88 \mathrm{~ms}$, settling 4810 nodes and relaxing 33481 edges. Further experiments on different metrics can be found in [26].

\subsection{An Even Larger Road Network}

Very recently, we obtained a new version of the European road network that is larger than the old one and covers more countries ${ }^{9}$. It has been provided for scientific use by the company ORTEC and consists of 33726989 nodes and 75108089 directed edges. We use the same parameters as for the old version (in particular, $H=30$ ) and observe a very good shrinking behaviour: we have 1.87 times as many nodes in the beginning, but after the construction of the same number of levels only 1.04 times as many nodes remain. Thus, the same number of levels is sufficient, only the distance table gets slightly bigger. We arrive at a preprocessing time of 18 minutes, a memory overhead of 37 bytes per node, and query times of $0.60 \mathrm{~ms}$ for random queries; on average, 685 nodes are settled and 2457 edges are relaxed.

\section{Discussion}

Highway hierarchies are a simple, robust and space-efficient concept that allows very efficient exact fastest-path queries even in huge real-world road networks. These attributes have been confirmed in an extensive experimental study. Although highway hierarchies are already very useful when applied directly, their usefulness extends to a much wider range: some concepts like the contraction of a network have turned out to be advantageous for other speedup techniques as well; they can be extended to deal with many-to-many queries; the currently fastest shortest-path algorithm for static road networks is based on them; an efficient approach to dealing with dynamic scenarios like traffic jams uses highway hierarchies in its preprocessing phase; ...

Nevertheless, a lot of interesting questions remain. How to handle mobile devices with limited fast memory? How to deal with multiple objective functions or with time-dependent edge weights? What about public transportation networks?

We are optimistic that highway hierarchies and related methods are a promising starting point to tackle several of these problems.

\section{References}

1. Dijkstra, E.W.: A note on two problems in connexion with graphs. Numerische Mathematik 1 (1959) 269-271

2. Sanders, P., Schultes, D.: Engineering fast route planning algorithms. In: 6th Workshop on Experimental Algorithms. Volume 4525 of LNCS., Springer (2007) 23-36

3. Hart, P.E., Nilsson, N.J., Raphael, B.: A formal basis for the heuristic determination of minimum cost paths. IEEE Transactions on System Science and Cybernetics 4 (1968) 100-107

4. Goldberg, A.V., Harrelson, C.: Computing the shortest path: $A^{*}$ meets graph theory. In: 16th ACM-SIAM Symposium on Discrete Algorithms. (2005) 156-165

5. Goldberg, A.V., Werneck, R.F.: An efficient external memory shortest path algorithm. In: Workshop on Algorithm Engineering and Experimentation. (2005) 26-40

\footnotetext{
${ }^{9}$ In addition to the old version, the Czech Republic, Finland, Hungary, Ireland, Poland, and Slovakia.
} 


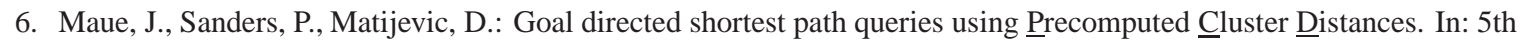
Workshop on Experimental Algorithms (WEA). Volume 4007 of LNCS., Springer (2006) 316-328

7. Schulz, F., Wagner, D., Weihe, K.: Dijkstra's algorithm on-line: An empirical case study from public railroad transport. In: 3rd Workshop on Algorithm Engineering. Volume 1668 of LNCS., Springer (1999) 110-123

8. Wagner, D., Willhalm, T.: Geometric speed-up techniques for finding shortest paths in large sparse graphs. In: 11th European Symposium on Algorithms. Volume 2832 of LNCS., Springer (2003) 776-787

9. Lauther, U.: An extremely fast, exact algorithm for finding shortest paths in static networks with geographical background. In: Geoinformation und Mobilität - von der Forschung zur praktischen Anwendung. Volume 22., IfGI prints, Institut für Geoinformatik, Münster (2004) 219-230

10. Köhler, E., Möhring, R.H., Schilling, H.: Acceleration of shortest path and constrained shortest path computation. In: 4th International Workshop on Efficient and Experimental Algorithms. (2005) 126-138

11. Möhring, R.H., Schilling, H., Schütz, B., Wagner, D., Willhalm, T.: Partitioning graphs to speed up Dijkstra's algorithm. In: 4th International Workshop on Efficient and Experimental Algorithms. (2005) 189-202

12. Köhler, E., Möhring, R.H., Schilling, H.: Fast point-to-point shortest path computations with arc-flags. In: 9th DIMACS Implementation Challenge [37]. (2006)

13. Lauther, U.: An experimental evaluation of point-to-point shortest path calculation on roadnetworks with precalculated edge-flags. In: 9th DIMACS Implementation Challenge [37]. (2006)

14. Thorup, M.: Compact oracles for reachability and approximate distances in planar digraphs. In: 42nd IEEE Symposium on Foundations of Computer Science. (2001) 242-251

15. Muller, L.F., Zachariasen, M.: Fast and compact oracles for approximate distances in planar graphs. In: 15th European Symposium on Algorithms. Volume 4698 of LNCS., Springer (2007) 657-668

16. Fakcharoenphol, J., Rao, S.: Planar graphs, negative weight edges, shortest paths, and near linear time. In: 42nd IEEE Symposium on Foundations of Computer Science. (2001) 232-241

17. Schulz, F., Wagner, D., Zaroliagis, C.D.: Using multi-level graphs for timetable information. In: 4th Workshop on Algorithm Engineering and Experiments. Volume 2409 of LNCS., Springer (2002) 43-59

18. Gutman, R.: Reach-based routing: A new approach to shortest path algorithms optimized for road networks. In: 6th Workshop on Algorithm Engineering and Experiments. (2004) 100-111

19. Goldberg, A., Kaplan, H., Werneck, R.: Reach for $A^{*}$ : Efficient point-to-point shortest path algorithms. In: Workshop on Algorithm Engineering \& Experiments, Miami (2006) 129-143

20. Goldberg, A., Kaplan, H., Werneck, R.: Better landmarks within reach. In: 6th Workshop on Experimental Algorithms. Volume 4525 of LNCS., Springer (2007) 38-51

21. Ishikawa, K., Ogawa, M., Azume, S., Ito, T.: Map Navigation Software of the Electro Multivision of the '91 Toyota Soarer. In: IEEE Int. Conf. Vehicle Navig. Inform. Syst. (1991) 463-473

22. Jagadeesh, G., Srikanthan, T., Quek, K.: Heuristic techniques for accelerating hierarchical routing on road networks. IEEE Transactions on Intelligent Transportation Systems 3 (2002) 301-309

23. Sanders, P., Schultes, D.: Highway hierarchies hasten exact shortest path queries. In: 13th European Symposium on Algorithms. Volume 3669 of LNCS., Springer (2005) 568-579

24. Sanders, P., Schultes, D.: Engineering highway hierarchies. In: 14th European Symposium on Algorithms. Volume 4168 of LNCS., Springer (2006) 804-816

25. Knopp, S., Sanders, P., Schultes, D., Schulz, F., Wagner, D.: Computing many-to-many shortest paths using highway hierarchies. In: Workshop on Algorithm Engineering and Experiments. (2007)

26. Delling, D., Sanders, P., Schultes, D., Wagner, D.: Highway hierarchies star. In: 9th DIMACS Implementation Challenge [37]. (2006)

27. Bast, H., Funke, S., Matijevic, D., Sanders, P., Schultes, D.: In transit to constant time shortest-path queries in road networks. In: Workshop on Algorithm Engineering and Experiments. (2007) 46-59

28. Bast, H., Funke, S., Sanders, P., Schultes, D.: Fast routing in road networks with transit nodes. Science 316 (2007) 566

29. Schultes, D., Sanders, P.: Dynamic highway-node routing. In: 6th Workshop on Experimental Algorithms. Volume 4525 of LNCS., Springer (2007) 66-79

30. Nannicini, G., Baptiste, P., Barbier, G., Krob, D., Liberti, L.: Fast paths in large-scale dynamic road networks. arXiv:0704.1068v2 [cs.NI] (2007)

31. Cormen, T.H., Leiserson, C.E., Rivest, R.L., Stein, C.: Introduction to Algorithms. 2nd edn. MIT Press (2001)

32. Skiena, S.S.: The Algorithm Design Manual. Springer (1998)

33. Schmid, W.: Berechnung kürzester Wege in Straßennetzen mit Wegeverboten. PhD thesis, Universität Stuttgart (2000)

34. Müller, K.: Berechnung kürzester Pfade unter Beachtung von Abbiegeverboten. Student Research Project, Universität Karlsruhe, supervised by F. Schulz and D. Wagner (2005)

35. U.S. Census Bureau, Washington, DC: UA Census 2000 TIGER/Line Files. http://www. census .gov/geo/ www/tiger/tigerua/ua_tgr2k.html (2002)

36. R Development Core Team: R: A Language and Environment for Statistical Computing. http://www. r-project.org (2004)

37. 9th DIMACS Implementation Challenge: Shortest Paths. http://www.dis.uniroma1.it/ challenge $9 /$ (2006) 


\section{A Query-Proof of Correctness}

Additional Notations. ' $\circ$ ' denotes path concatenation. $\operatorname{succ}(u, P)$ and $\operatorname{pred}(u, P)$ denote the direct successor and predecessor of $u$ on $P$, respectively. We just write $\operatorname{succ}(u)$ and $\operatorname{pred}(u)$ if the path is clear from the context. For two nodes $u$ and $v$ on some path, $\min (u, v)$ denotes $u$ if $u \preceq v$ and $v$ otherwise. $\max (u, v)$ is defined analogously. $d_{P}(u, v):=w\left(\left.P\right|_{u \rightarrow v}\right)$ denotes the distance from $u$ to $v$ along the path $P$. Note that for any edge $(u, v)$ on $P$, we have $w(u, v)=d_{P}(u, v)$.

\section{A.1 Termination and Special Cases}

Since we have set the neighbourhood radius in the topmost level to infinity (R2), we are never tempted to go upwards beyond the topmost level. This observation is formalised in the following lemma.

Lemma 2. The for-loop in Line 9 of the highway query algorithm always terminates with $\ell \leq L$ and $(\ell=L \rightarrow$ gap $=\infty)$.

Proof. We only consider iterations where the forward search direction is selected; analogous arguments apply to the backward direction. By an inductive proof, we show that at the beginning of any iteration of the main while-loop, we have $\ell(u) \leq L$ and $(\ell(u)=L \rightarrow$ $\operatorname{gap}(u)=\infty$ ) for any node $u$ in $\vec{Q}$.

Base Case: True for the first iteration, where only $s$ belongs to $\vec{Q}$ : we have $\ell(s)=0 \leq L$ and $\operatorname{gap}(s)=r_{0}(s)$ (Line 2), which is equal to infinity if $L=0$ (due to R2).

Induction Step: We assume that our claim is true for iteration $i$ and show that it also holds for iteration $i+1$. Due to the induction hypothesis, we have $\ell(u) \leq L$ and $(\ell(u)=L \rightarrow$ $\operatorname{gap}(u)=\infty$ ) in Line 5. If $\ell(u)=L$, we have gap $=\operatorname{gap}^{\prime}=\underset{\ell(u)}{\vec{\ell}}(u)=\infty$ (Line 7 and 9, R2); thus the for-loop in Line 9 terminates immediately with $\ell=\ell(u)=L$. Otherwise $(\ell(u)<L)$, the for-loop either terminates with $\ell<L$ or reaches $\ell=L$; in the latter case, we have gap $=r_{\ell}(u)=\infty$ (Line 9, R2); hence, the loop terminates.

Thus, in any case, the loop terminates with $\ell \leq L$ and $(\ell=L \rightarrow$ gap $=\infty)$. Therefore, if the node $v$ adopts the key $k$ in Line 13 (either by a decreaseKey or an insert operation), the new key fulfils the required condition.

This concludes our inductive proof, which also shows that the claim of this lemma holds during any iteration of the main while-loop.

It is easy to the see that the following property of Dijkstra's algorithm also holds for the highway query algorithm.

Proposition 1. For each search direction, the sequence of distances $\delta(u)$ of settled nodes $u$ is monotonically increasing.

Now, we can prove that

Lemma 3. The highway query algorithm terminates.

Proof. The for-loop in Line 9 always terminates due to Lemma 2. The for-loop in Line 8 terminates since the edge set is finite. The main while-loop in Line 3 terminates since each node $v$ is inserted into each priority queue at most once, namely if it is unreached (Line 13); if it is reached, it either already belongs to the priority queue or it has already been settled; in the latter case, we know that $\delta(v) \leq \delta(u) \leq \delta(u)+w(e)$ (Proposition 1; edge weights are nonnegative) so that no priority queue operation is performed due to the specification of the decreaseKey operation. 
The special case that there is no path from $s$ to $t$ is trivial. The algorithm terminates due to Lemma 3 and returns $\infty$ since no node can be settled from both search directions (otherwise, there would be some path from $s$ to $t$ ). For the remaining proof, we assume that a shortest path from $s$ to $t$ exists in the original graph $G$.

\section{A.2 Contracted and Expanded Paths}

Lemma 4. Shortcuts do not overlap, i.e., if there are four nodes $u \prec u^{\prime} \prec v \prec v^{\prime}$ on a path $P$ in $G$, then there cannot exist both a shortcut $(u, v)$ and a shortcut $\left(u^{\prime}, v^{\prime}\right)$ at the same time.

Proof. Let us assume that there is a shortcut $(u, v) \in S_{\ell}$ for some level $\ell$. All inner nodes, in particular $u^{\prime}$, belong to $B_{\ell}$. Since $u^{\prime}$ does not belong to $V_{\ell}^{\prime}$, a shortcut that starts from $u^{\prime}$ can belong only to some level $k<\ell$. If there was a shortcut $\left(u^{\prime}, v^{\prime}\right) \in S_{k}$, the inner node $v$ would have to belong to $B_{k}$, which is a contradiction since $v \in V_{\ell}^{\prime}$.

Definition 1. For a given path $P$ in a given highway hierarchy $\mathcal{G}$, the contracted path $\operatorname{ctr}(P)$ is defined in the following way: while there is a subpath $\left\langle u, b_{1}, b_{2}, \ldots, b_{k}, v\right\rangle$ with $u, v \in V_{\ell}^{\prime}$ and $b_{i} \in B_{\ell}, 1 \leq i \leq k, k \geq 1$, for some level $\ell$, replace it by the shortcut edge $(u, v) \in S_{\ell}$.

Note that this definition terminates since the number of nodes in the path is reduced by at least one in each step and the definition is unambiguous due to Lemma 4.

Definition 2. For a given path $P$ in a given highway hierarchy $\mathcal{G}$, the level- $\ell$ expanded path $\exp (P, \ell)$ is defined in the following way: while the path contains a shortcut edge $(u, v) \in S_{k}$ for some $k>\ell$, replace it by the represented path in $G_{k}$.

Note that this definition terminates since an expanded subpath can only contain shortcuts of a smaller level.

\section{A.3 Highway Path}

Consider a given highway hierarchy $\mathcal{G}$ and an arbitrary path $P=\langle s, \ldots, t\rangle$. In the following, we will bring out the structure of $P$ w.r.t. $\mathcal{G}$.

Last Neighbour and First Core Node. For any level $\ell$ and any node $u$ on $P$, we define the last succeeding level- $\ell$ neighbour $\vec{\omega}_{\ell}^{P}(u)$ and the first succeeding level- $\ell$ core node $\vec{\alpha}_{\ell}^{P}(u)$ : $\vec{\omega}_{\ell}^{P}(u)$ is the node $v \in\left\{x \in P \mid u \preceq x \wedge d_{P}(u, x) \leq r_{\ell}(u)\right\}$ that maximises $d_{P}(u, v)$, and $\vec{\alpha}_{\ell}^{P}(u)$ is the node $v \in\{t\} \cup\left\{x \in P \cap V_{\ell}^{\prime} \mid u \preceq x\right\}$ that minimises $d_{P}(u, v)$. The last preceding neighbour $\overleftarrow{\omega}_{\ell}^{P}(u)$ and the first preceding core node $\overleftarrow{\alpha}_{\ell}^{P}(u)$ are defined analogously.

Unidirectional Labelling. Now, we inductively define a forward labelling of the path $P$. The labels $s_{0}$ and $s_{0}^{\prime}$ are set to $s$ and for $\ell, 0 \leq \ell<L$, we set $s_{\ell+1}:=\vec{\omega}_{\ell}^{P}\left(s_{\ell}^{\prime}\right)$ and $s_{\ell+1}^{\prime}:=\vec{\alpha}_{\ell+1}^{P}\left(s_{\ell+1}\right)$. Furthermore, in order to avoid some case distinctions, $s_{L+1}:=t$. For an example, we refer to Figure 9.

Proposition 2. The following properties apply to the (Unidirectional) forward labelling of $P$ :

- U1: $s=s_{0}=s_{0}^{\prime} \preceq s_{1} \preceq s_{1}^{\prime} \preceq \ldots \preceq s_{L} \preceq s_{L}^{\prime} \preceq s_{L+1}=t$

- U2a: $\forall \ell, 0 \leq \ell \leq L: \forall u, s_{\ell}^{\prime} \preceq u \preceq s_{\ell+1}: d_{P}\left(s_{\ell}^{\prime}, u\right) \leq r_{\ell}\left(s_{\ell}^{\prime}\right)$

- U2b: $\forall \ell, 0 \leq \ell \leq L: \forall u \succ s_{\ell+1}: d_{P}\left(s_{\ell}^{\prime}, u\right)>r_{\ell}\left(s_{\ell}^{\prime}\right)$

- U3: $\forall \ell, 0 \leq \ell \leq L: \forall u, s_{\ell} \preceq u \prec s_{\ell}^{\prime}: u \notin V_{\ell}^{\prime}$

- U4: $\forall \ell, 0 \leq \ell \leq L: s_{\ell}^{\prime}=t \vee s_{\ell}^{\prime} \in V_{\ell}^{\prime}$

A backward labelling (specifying nodes $t_{\ell}$ and $t_{\ell}^{\prime}$ ) is defined analogously. 
Meeting Level and Point. The meeting level $\lambda$ of $P$ is 0 if $s=t$ and $\max \left\{\ell \mid s_{\ell} \preceq t_{\ell}\right\}$ if $s \neq t$. Note that $\lambda \leq L$ (in any case) and $t_{\lambda+1} \prec s_{\lambda+1}$ (in case that $s \neq t$ ). The meeting point $p$ of $P$ is either $t_{\lambda}$ (if $t_{\lambda} \preceq s_{\lambda}^{\prime}$ ) or $\min \left(s_{\lambda+1}, t_{\lambda}^{\prime}\right)$ (otherwise). Figure 10 gives an example.

Proposition 3. The following properties apply to the Meeting point $p$ of $P$ :

- M1: $s_{\lambda} \preceq p \preceq t_{\lambda}$

- M2: $t_{\lambda+1} \preceq p \preceq s_{\lambda+1}$

- M3: $\forall \ell, 0 \leq \ell \leq L:\left(s_{\ell}^{\prime} \prec p \rightarrow p \preceq t_{\ell}^{\prime}\right) \wedge\left(p \prec t_{\ell}^{\prime} \rightarrow s_{\ell}^{\prime} \preceq p\right)$

Proof. The case $s=t$ is trivial. Subsequently, we assume $s \neq t$. In order to prove M1, M2, and (M3 for $\ell=\lambda$ ), we distinguish between two cases.

Case 1: $t_{\lambda} \preceq s_{\lambda}^{\prime}$. Then, $p=t_{\lambda}$. M1 is fulfilled due to the definition of the meeting level, which implies $s_{\lambda} \preceq t_{\lambda}$. Furthermore, due to U1, we have $t_{\lambda+1} \preceq t_{\lambda}^{\prime} \preceq t_{\lambda}=p \preceq s_{\lambda}^{\prime} \preceq s_{\lambda+1}$ so that M2 and (M3 for $\ell=\lambda$ ) are fulfilled.

Case 2: $s_{\lambda}^{\prime} \prec t_{\lambda}$. Then, $p=\min \left(s_{\lambda+1}, t_{\lambda}^{\prime}\right)$.

Subcase 2.1: $s_{\lambda+1} \preceq t_{\lambda}^{\prime}$. Then, $p=s_{\lambda+1}$. We have $s_{\lambda} \preceq s_{\lambda}^{\prime} \preceq s_{\lambda+1}=p \preceq t_{\lambda}^{\prime} \preceq t_{\lambda}$ so that M1 and (M3 for $\ell=\lambda$ ) are fulfilled. Furthermore, M2 holds due to $t_{\lambda+1} \prec s_{\lambda+1}$.

Subcase 2.2: $t_{\lambda}^{\prime} \prec s_{\lambda+1}$. Then, $p=t_{\lambda}^{\prime}$. Since $s_{\lambda}^{\prime} \prec t_{\lambda} \preceq t$, we know that $s_{\lambda}^{\prime} \in V_{\lambda}^{\prime}$ (due to U4). Thus, we have $s_{\lambda}^{\prime} \preceq t_{\lambda}^{\prime} \preceq t_{\lambda}$ (otherwise $\left(t_{\lambda}^{\prime} \prec s_{\lambda}^{\prime} \preceq t_{\lambda}\right.$ ), we would have a contradiction with U3). Hence, $s_{\lambda} \preceq s_{\lambda}^{\prime} \preceq t_{\lambda}^{\prime}=p \preceq t_{\lambda}$ so that M1 and (M3 for $\ell=\lambda$ ) are fulfilled. M2 holds as well since $t_{\lambda+1} \preceq t_{\lambda}^{\prime}=p \prec s_{\lambda+1}$.

It remains to show M3 for $\ell<\lambda$ and for $\ell>\lambda$. In the former case, M3 holds due to M1, which implies $s_{\ell}^{\prime} \preceq s_{\lambda} \preceq p \preceq t_{\lambda} \preceq t_{\ell}^{\prime}$ (U1). In the latter case, M3 holds due to M2, which implies $t_{\ell}^{\prime} \preceq t_{\lambda+1} \preceq p \preceq s_{\lambda+1} \preceq s_{\ell}^{\prime}$ (U1).

Highway Path. $P=\langle s, \ldots, t\rangle$ is a highway path (Figure 11) iff the following two Highway properties are fulfilled:

- $\mathrm{H} 1: \forall \ell, 0 \leq \ell \leq L: \mathrm{H} 1(\ell)$

- $\mathrm{H} 2: \forall \ell, 0 \leq \ell \leq L: \mathrm{H} 2(\ell)$

where

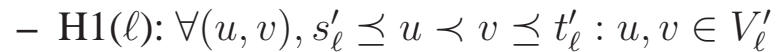

- $\mathrm{H} 2(\ell): \forall(u, v), s_{\ell} \preceq u \prec v \preceq t_{\ell}: \ell(u, v) \geq \ell$

\section{A.4 Reachability Along a Highway Path}

We consider a path $P=\langle s, \ldots, t\rangle$. For a node $u$ on $P$, we define the reference level $\bar{\ell}(u):=$ $\max \left(\{0\} \cup\left\{i \mid s_{i} \prec u\right\}\right)$.

Proposition 4. For any two nodes $u$ and $v$ with $u \preceq v$, the following reference Level properties apply:

- L1: $0 \leq \bar{\ell}(u) \leq L$

- L2: $s_{\bar{\ell}(u)} \preceq u$

- L3: $u \preceq s_{\bar{\ell}(u)+1}$

- L4: $\bar{\ell}(u) \leq \bar{\ell}(v)$

Definition 3. A node $u$ is said to be Appropriately reached/settled with the key $k=(\delta(u)$, $\ell(u)$, gap $(u))$ on the path $P$ iff all of the following conditions are fulfilled:

- $A_{1}(k, u): \delta(u)=d_{0}(s, u)$ 
- $A_{2}(k, u): \ell(u)=\bar{\ell}(u)$

- $A_{3}(k, u): \operatorname{gap}(u)= \begin{cases}\infty & \text { if } u \preceq s_{\ell(u)}^{\prime} \\ r_{\ell(u)}\left(s_{\ell(u)}^{\prime}\right)-d_{P}\left(s_{\ell(u)}^{\prime}, u\right) & \text { otherwise }\end{cases}$
- $A_{4}(u): \forall i: t \neq s_{i}^{\prime} \preceq u \longrightarrow u \in V_{i}^{\prime}$

The following (somewhat technical) lemma will be used to prove Lemmas 6 and 7. Basically, it states that in the highway query algorithm the search level and the gap to the next applicable neighbourhood border are set correctly.

Lemma 5. Consider a path $P=\langle s, \ldots, t\rangle$ and an edge $(u, v)$ on $P$. Assume that $u$ is settled by the highway query algorithm appropriately with some key $k$. We consider the attempt to relax the edge $(u, v)$. After Line 9 has been executed, the following Invariants apply w.r.t. the variables $\ell$ and gap:

- I1: (a) $s_{\ell} \preceq u \wedge$ (b) $v \preceq s_{\ell+1}$

$-\mathrm{I} 2: \ell=\bar{\ell}(v)$

- I3: gap $= \begin{cases}\infty & \text { if } v \preceq s_{\ell}^{\prime}, \\ r_{\ell}\left(s_{\ell}^{\prime}\right)-d_{P}\left(s_{\ell}^{\prime}, u\right) & \text { otherwise. }\end{cases}$

Proof. We distinguish between two cases in order to prove I1 and I3.

Case 1: zero iterations of the for-loop in Line 9 take place $(\ell=\ell(u)$ ).

In this case, we have $\ell=\ell(u)$ and $w(u, v) \leq$ gap'. Hence, $s_{\ell} \preceq u$ due to $A_{2}(k, u)$ and L2 $(\Rightarrow$ I1a). In order to show I1b and I3, we distinguish between three subcases:

- Subcase 1.1: $u \prec s_{\ell}^{\prime} \Rightarrow v \preceq s_{\ell}^{\prime} \preceq s_{\ell+1}$ (U1) ( $\Rightarrow$ I1b). Furthermore, because of gap $(u)=$ $\infty\left(A_{3}(u, k)\right)$, we have gap $=$ gap $^{\prime}=r_{\ell(u)}(u)=\infty$ due to U3 and R1 $(\Rightarrow$ I3 since $\left.v \preceq s_{\ell}^{\prime}\right)$.

- Subcase 1.2: $u=s_{\ell}^{\prime} \Rightarrow \operatorname{gap}(u)=\infty\left(A_{3}(u, k)\right) \Rightarrow w(u, v) \leq \operatorname{gap}^{\prime}=r_{\ell}(u)$ (Line 7) $\Rightarrow d_{P}\left(s_{\ell}^{\prime}, v\right) \leq r_{\ell}\left(s_{\ell}^{\prime}\right)$ (since $\left.u=s_{\ell}^{\prime}\right) \Rightarrow v \preceq s_{\ell+1}$ (U2b) $(\Rightarrow$ I1b). Furthermore, gap $=$ $\operatorname{gap}^{\prime}=r_{\ell}(u)=r_{\ell}\left(s_{\ell}^{\prime}\right)-d_{P}\left(s_{\ell}^{\prime}, u\right)$ (since $u=s_{\ell}^{\prime}$ ) implies I3 since $s_{\ell}^{\prime} \prec v$.

- Subcase 1.3: $u \succ s_{\ell}^{\prime} \Rightarrow \operatorname{gap}(u)=r_{\ell}\left(s_{\ell}^{\prime}\right)-d_{P}\left(s_{\ell}^{\prime}, u\right)\left(A_{3}(u, k)\right)$. By Lemma $2, \ell \leq L$ and $(\ell=L \rightarrow$ gap $=\infty)$. If $\ell=L$, we have $v \preceq t=s_{L+1}=s_{\ell+1}$ ( $\Rightarrow$ I1b) and gap $=\infty=r_{\ell}\left(s_{\ell}^{\prime}\right)-d_{P}\left(s_{\ell}^{\prime}, u\right)(\mathrm{R} 2)\left(\Rightarrow\right.$ I3 since $\left.s_{\ell}^{\prime} \prec v\right)$. Subsequently, we deal with the remaining case $\ell<L$. The facts that $u \preceq t$ and $s_{\ell}^{\prime} \prec u$ imply $s_{\ell}^{\prime} \neq t$, which yields $s_{\ell}^{\prime} \in V_{\ell}^{\prime}$ due to U4. Hence, due to R3, $\operatorname{gap}(u) \neq \infty \Rightarrow w(u, v) \leq \operatorname{gap}^{\prime}=\operatorname{gap}(u)$ (Line 7) $\Rightarrow d_{P}(u, v) \leq r_{\ell}\left(s_{\ell}^{\prime}\right)-d_{P}\left(s_{\ell}^{\prime}, u\right) \Rightarrow d_{P}\left(s_{\ell}^{\prime}, v\right) \leq r_{\ell}\left(s_{\ell}^{\prime}\right) \Rightarrow v \preceq s_{\ell+1}$ (U2b) ( $\Rightarrow$ I1b). Furthermore, gap $=$ gap $^{\prime}=\operatorname{gap}(u)=r_{\ell}\left(s_{\ell}^{\prime}\right)-d_{P}\left(s_{\ell}^{\prime}, u\right)$ implies I3 since $s_{\ell}^{\prime} \prec v$.

Case 2: at least one iteration of the for-loop takes place $(\ell>\ell(u))$.

We claim that after any iteration of the for-loop, we have $u=s_{\ell}$. Proof by induction:

Base Case: We consider the first iteration of the for-loop. Line 9 and the fact that an iteration takes place imply $w(u, v)>$ gap $^{\prime}$, which means that gap ${ }^{\prime} \neq \infty$. We distinguish between two subcases to show that $d_{P}\left(s_{\ell(u)}^{\prime}, v\right)>\underset{\ell(u)}{\overrightarrow{r_{\ell}}}\left(s_{\ell(u)}^{\prime}\right)$.

- Subcase 2.1: $u \preceq s_{\ell(u)}^{\prime} \Rightarrow \operatorname{gap}(u)=\infty\left(A_{3}(u, k)\right) \Rightarrow w(u, v)>\operatorname{gap}^{\prime}=r_{\ell(u)}(u)$ (Line 7) $\Rightarrow r_{\ell(u)}(u) \neq \infty$. We have $s_{\ell(u)} \preceq u \preceq s_{\ell(u)}^{\prime}$ due to L2, $A_{2}(u, k)$, and the assumption of Subcase 2.1. However, we can exclude that $s_{\ell(u)} \preceq u \prec s_{\ell(u)}^{\prime}$ : this would imply $u \notin V_{\ell(u)}^{\prime}$ (U3) and, thus, $r_{\ell(u)}(u)=\infty$ (R1). Therefore, $u=s_{\ell(u)}^{\prime} \Rightarrow d_{P}\left(s_{\ell(u)}^{\prime}, v\right)>r_{\ell(u)}\left(s_{\ell(u)}^{\prime}\right)$

- Subcase 2.2: $u \succ s_{\ell(u)}^{\prime} \Rightarrow s_{\ell(u)}^{\prime} \neq t \Rightarrow s_{\ell(u)}^{\prime} \in V_{\ell(u)}^{\prime}$ (U4). Furthermore, gap $(u)=$ $r_{\ell(u)}\left(s_{\ell(u)}^{\prime}\right)-d_{P}\left(s_{\ell(u)}^{\prime}, u\right)\left(A_{3}(u, k)\right) \Rightarrow \operatorname{gap}(u) \neq \infty($ due to R3 since $\ell(u)<L$ (Lemma 2) and $\left.s_{\ell(u)}^{\prime} \in V_{\ell(u)}^{\prime}\right) \Rightarrow d_{P}(u, v)=w(u, v)>\operatorname{gap}^{\prime}=\operatorname{gap}(u)=\underset{r_{\ell(u)}}{\left(s_{\ell(u)}^{\prime}\right)}-d_{P}\left(s_{\ell(u)}^{\prime}, u\right)$ (Line 7) $\Rightarrow d_{P}\left(s_{\ell(u)}^{\prime}, v\right)>r_{\ell(u)}\left(s_{\ell(u)}^{\prime}\right)$ 
From $d_{P}\left(s_{\ell(u)}^{\prime}, v\right)>\overrightarrow{r_{\ell(u)}}\left(s_{\ell(u)}^{\prime}\right)$, it follows that $s_{\ell(u)+1} \prec v(\mathrm{U} 2 \mathrm{a})$, which implies $s_{\ell(u)+1} \preceq u$. Hence, $u=s_{\ell(u)+1}$ (since $u \preceq s_{\ell(u)+1}$ due to L3 and $A_{2}(k, u)$ ).

Induction Step: Let us now deal with the iteration from level $i$ to level $i+1$ for $i \geq \ell(u)+1$. We have $w(u, v)>$ gap $=r_{i}(u)$, which implies $r_{i}(u) \neq \infty$. Starting with $u=s_{i} \preceq$ $s_{i}^{\prime} \preceq s_{i+1}$ (induction hypothesis, U1), we can conclude that $u=s_{i}^{\prime}(\mathrm{U} 3, \mathrm{R} 1) \Rightarrow d_{P}\left(s_{i}^{\prime}, v\right)>$ $\left.r_{i} \overrightarrow{(} s_{i}^{\prime}\right) \Rightarrow s_{i+1} \prec v(\mathrm{U} 2 \mathrm{a}) \Rightarrow s_{i+1} \preceq u \Rightarrow u=s_{i+1}$ (since $u \preceq s_{i+1}$ ). This completes our inductive proof.

After the last iteration, we have $u=s_{\ell} \preceq s_{\ell}^{\prime}$ ( $\Rightarrow$ I1a). Furthermore, $w(u, v) \leq r_{\ell}(u)$. If $u \prec s_{\ell}^{\prime}$, we obtain $v \preceq s_{\ell}^{\prime} \preceq s_{\ell+1}$ ( $\Rightarrow$ I1b) and gap $=r_{\ell}(u)=\infty$ due to U3 and R1 ( $\Rightarrow$ I3 since $\left.v \preceq s_{\ell}^{\prime}\right)$. Otherwise $\left(u=s_{\ell}^{\prime}\right)$, we get $d_{P}\left(s_{\ell}^{\prime}, v\right) \leq r_{\ell}\left(s_{\ell}^{\prime}\right)$, which implies $v \preceq s_{\ell+1}$ as well (U2b) ( $\Rightarrow$ I1b); furthermore, gap $=r_{\ell}(u)=r_{\ell}\left(s_{\ell}^{\prime}\right)-d_{P}\left(s_{\ell}^{\prime}, u\right)$ (since $u=s_{\ell}^{\prime}$ ) implies I3 since $s_{\ell}^{\prime} \prec v$. This completes the proof of I1 and I3.

I2 $(\bar{\ell}(v)=\ell)$ directly follows from $s_{\ell} \prec v \preceq s_{\ell+1}$ (I1).

Lemma 6. Consider a highway path $P=\langle s, \ldots, t\rangle$ and an edge $(u, v)$ on $P$ such that $u$ precedes the meeting point $p$. Assume that $u$ has been appropriately settled. Then, the edge $(u, v)$ is not skipped, but relaxed.

Proof. We consider the relaxation of the edge $(u, v)$. Due to Lemma 5, the Invariants I1I3 apply after Line 9 has been executed. Now, we consider Line 10 of the highway query algorithm.

I1 and M2 imply $s_{\ell} \preceq u \prec p \preceq s_{\lambda+1}$. Hence, $\ell \leq \lambda$. Thus, $u \prec p \preceq t_{\lambda} \preceq t_{\ell}$ (M1). By $\mathrm{H} 2$, we obtain $\ell(u, v) \geq \ell$. Therefore, the edge $(u, v)$ is not skipped at this point.

Moreover, we prove that the condition in Line 11 is not fulfilled since $(u, v)$ belongs to a highway path. This means that the edge $(u, v)$ is not skipped at this point, either. We have to show that $u \notin V_{\ell}^{\prime} \vee v \notin B_{\ell}$. We have $s_{\ell} \preceq u$ (I1). If $u \prec s_{\ell}^{\prime}$, we get $u \notin V_{\ell}^{\prime}$ (U3). Otherwise, we have $s_{\ell}^{\prime} \preceq u \prec v \preceq p \preceq t_{\ell}^{\prime}$ (M3), which yields $v \notin B_{\ell}$ (H1).

Therefore, $(u, v)$ is not skipped, but relaxed.

Lemma 7. Consider a shortest path $P=\langle s, \ldots, t\rangle$ and an edge $(u, v)$ on $P$. Assume that $u$ has been appropriately settled with some key $k$. Furthermore, assume that the edge $(u, v)$ is not skipped, but relaxed. Then, $v$ can be appropriately reached from $u$ with key $k^{\prime}$.

Proof. We consider the relaxation of the edge $(u, v)$. Due to Lemma 5, the Invariants I1-I3 apply after Line 9 has been executed. Therefore-since $(u, v)$ is not skipped, but relaxed-, the node $v$ can be reached with the key

$$
k^{\prime}=\left(\delta^{\prime}(v), \ell^{\prime}(v), \operatorname{gap}^{\prime}(v)\right):=(\delta(u)+w(u, v), \ell, \operatorname{gap}-w(u, v)) .
$$

Thus, $A_{1}\left(k^{\prime}, v\right), A_{2}\left(k^{\prime}, v\right)$, and $A_{3}\left(k^{\prime}, v\right)$ hold since $P$ is a shortest path and due to $A_{1}(k, u)$, $\mathrm{I} 2$, and $\mathrm{I} 3$.

Consider an arbitrary $i$ such that $t \neq s_{i}^{\prime} \preceq v$. To prove $A_{4}(v)$, we have to show that $v \in V_{i}^{\prime}$. Due to $\mathrm{U} 4$, this is true for $s_{i}^{\prime}=v$. Now, we deal with the remaining case $s_{i}^{\prime} \preceq u \prec v$. Since $v \preceq s_{\ell+1} \preceq s_{\ell+1}^{\prime}$ (I1, U1), we have $i \leq \ell$. The case $\ell=0$ is trivial; hence, we assume $\ell>0$. Since the edge $(u, v)$ is not skipped, we know that Restriction 1 does not apply. Therefore, we have $\ell(u, v) \geq \ell$, which implies $v \in V_{\ell} \subseteq V_{\ell-1}^{\prime}$. For $i<\ell$, we have $V_{\ell-1}^{\prime} \subseteq V_{i}^{\prime}$ and are done. For $i=\ell$, we have $u \in V_{\ell}^{\prime}$ due to $A_{4}(u)$. This implies $v \notin B_{\ell}$ since Restriction 2 does not apply as well. $v \in V_{\ell}$ and $v \notin B_{\ell}$ yield $v \in V_{\ell}^{\prime}$.

Analogous considerations hold for the backward search. 


\section{A.5 Finding an Optimal Path}

Source and target nodes $s$ and $t$ are given such that a shortest path from $s$ to $t$ exists. ${ }^{10}$

Definition 4. A state $z$ is a triple $(P, u, \bar{u})$, where $P$ is a s-t-path, $u, \bar{u} \in V \cap P$, and $u \preceq \bar{u}$.

Definition 5. A state $z=(P, u, \bar{u})$ is valid iff all of the following valid State properties are fulfilled:

- S1: $w(P)=d_{0}(s, t)$

- S2: $\left.P\right|_{u \rightarrow \bar{u}}$ is contracted, i.e., $\left.P\right|_{u \rightarrow \bar{u}}=\operatorname{ctr}\left(\left.P\right|_{u \rightarrow \bar{u}}\right)$

- S3: $\left.P\right|_{s \rightarrow u}$ and $\left.P\right|_{\bar{u} \rightarrow t}$ are paths in the forward and backward search tree, respectively.

Lemma 8. Consider a valid state $z=(P, u, \bar{u})$ and an arbitrary node $x, s \preceq x \preceq u$, on $P$. Then, $x$ has been appropriately settled. Analogously for backward search.

Proof. Base Case: True for s. Induction Step: We assume that $y, s \preceq y \prec u$, has been appropriately settled and show that $x=\operatorname{succ}(y)$ is appropriately settled as well. Since $(y, x)$ belongs to the forward search tree (S3), we know that $(y, x)$ is not skipped, but relaxed. The other prerequisites of Lemma 7 are fulfilled as well (due to the induction hypothesis and S1). Thus, we can conclude that $x$ can be appropriately reached from $y$. Since $(y, x)$ belongs to the forward search tree, we know that $x$ is also settled from $y$.

Lemma 9. If $z=(P, u, \bar{u})$ is a valid state, then $P$ is a highway path.

Proof. All labels (e.g., $s_{\ell}^{\prime}$ ) in this proof refer to $P$. We show that the highway properties H1 and $\mathrm{H} 2$ are fulfilled by induction over the level $\ell$.

Base Case: $\mathrm{H} 2(0)$ trivially holds since $\ell(u, v) \geq 0$ for any edge $(u, v)$.

Induction Step $(a): \mathrm{H} 2(\ell) \rightarrow \mathrm{H} 1(\ell)$. We assume $s_{\ell}^{\prime} \prec t_{\ell}^{\prime}$. (Otherwise, $\mathrm{H} 1(\ell)$ is trivially fulfilled.) This implies $s_{\ell}^{\prime} \neq t$. Consider an arbitrary node $x$ on $\left.P\right|_{s_{\ell}^{\prime} \rightarrow t_{\ell}^{\prime}}$. We distinguish between three cases.

Case 1: $x \preceq u$. According to Lemma $8, A_{4}(x)$ holds. Hence, $x \in V_{\ell}^{\prime}$ since $s_{\ell}^{\prime} \preceq x$.

Case 2: $u \preceq x \preceq \bar{u}$. We have $y:=\max \left(u, s_{\ell}^{\prime}\right) \in V_{\ell}^{\prime}$ (either by Lemma 8: $A_{4}(u)$ or by U4). Analogously, $\bar{y}:=\min \left(\bar{u}, t_{\ell}^{\prime}\right) \in V_{\ell}^{\prime}$. Since $u \preceq y \preceq x \preceq \bar{y} \preceq \bar{u}$ and $\left.P\right|_{u \rightarrow \bar{u}}=\operatorname{ctr}\left(\left.P\right|_{u \rightarrow \bar{u}}\right)$ (S2), we can conclude that $x \notin B_{\ell}$. Furthermore, we have $x \in V_{\ell}$ (due to H2( $\ell$ )). Thus, $x \in V_{\ell}^{\prime}$.

Case 3: $\bar{u} \preceq x$. Analogous to Case 1 .

Induction Step $(b): \mathrm{H} 1(\ell) \wedge \mathrm{H} 2(\ell) \rightarrow \mathrm{H} 2(\ell+1)$. Let $\bar{P}$ denote $\exp \left(\left.P\right|_{s_{\ell}^{\prime} \rightarrow t_{\ell}^{\prime}}, \ell\right)$ and consider an arbitrary edge $(x, y)$ on $\bar{P}$. If $(x, y)$ is part of an expanded shortcut, we have $\ell(x, y) \geq \ell+1$ and $x, y \in V_{\ell+1} \subseteq V_{\ell}^{\prime}$. Otherwise, $(x, y)$ belongs to $\left.P\right|_{s_{\ell}^{\prime} \rightarrow t_{\ell}^{\prime}}$, which is a subpath of $\left.P\right|_{s_{\ell \rightarrow t_{\ell}}}$, which implies $x, y \in V_{\ell}^{\prime}$ and $\ell(x, y) \geq \ell$ by $\mathrm{H} 1(\ell)$ and $\mathrm{H} 2(\ell)$. Thus, in any case, $\ell(x, y) \geq \ell$, $x, y \in V_{\ell}^{\prime}$, and $(x, y)$ is not a shortcut of some level $>\ell$. Hence, $\bar{P}$ is a path in $G_{\ell}^{\prime}$. Now, consider an arbitrary edge $(u, v), s_{\ell+1} \preceq u \prec v \preceq t_{\ell+1}$, on $P$. If $(u, v)$ is a shortcut of some level $>\ell$, we directly have $\ell(u, v) \geq \ell+1$. Otherwise, $(u, v)$ is on $\bar{P}$ as well. Since $s_{\ell+1} \prec v$, we have $d_{P}\left(s_{\ell}^{\prime}, v\right)>r_{\ell}\left(s_{\ell}^{\prime}\right)$ (U2b). Moreover, $\mathrm{S} 1$ implies that $\bar{P}$ is a shortest path in $G_{\ell}^{\prime}$ and, in particular, $d_{\bar{P}}\left(s_{\ell}^{\prime}, v\right)=w\left(\left.\bar{P}\right|_{s_{\ell}^{\prime} \rightarrow v}\right)=d_{\ell}\left(s_{\ell}^{\prime}, v\right)$. Using the fact that $d_{\bar{P}}\left(s_{\ell}^{\prime}, v\right)=d_{P}\left(s_{\ell}^{\prime}, v\right)$, we obtain $d_{\ell}\left(s_{\ell}^{\prime}, v\right)>r_{\ell}\left(s_{\ell}^{\prime}\right)$ and, thus, $v \notin \mathcal{N}_{\ell} \rightarrow\left(s_{\ell}^{\prime}\right)$.

Analogously, we have $u \notin \mathcal{N}_{\ell}^{\leftarrow}\left(t_{\ell}^{\prime}\right)$. Hence, the definition of the highway network $G_{\ell+1}$ implies $(u, v) \in E_{\ell+1}$. Thus, $\ell(u, v) \geq \ell+1$.

Definition 6. A valid state is either a final state (if $u=\bar{u}$ ) or a non-final state (otherwise).

\footnotetext{
${ }^{10}$ The special case that there is no path from $s$ to $t$ is treated in Section A.1.
} 
We pick any shortest $s$-t-path $P$. The state $(\operatorname{ctr}(P), s, t)$ is the initial state. Since forward and backward search run completely independently of each other, any serialisation of both search processes will yield exactly the same result. Therefore, in our proof, we are free to pick-w.l.o.g. - any order of forward and backward steps. We assume that at first one forward and one backward iteration is performed, which implies that $s$ and $t$ are settled. At this point, the highway query algorithm is in the initial state. It is easy to see that the initial state is a valid state. Due to the following lemma, it is sufficient to prove that a final state is eventually reached.

Lemma 10. Getting to a final state is equivalent to finding a shortest s-t-path.

Proof. $u=\bar{u}$ means that forward and backward search meet. Due to Lemma 8, we can conclude that both $u$ and $\bar{u}$ are settled with the optimal distance $\left(A_{1}\right)$, i.e., $\vec{\delta}(u)=d_{0}(s, u)$ and $\overleftarrow{\delta}(\bar{u})=d_{0}(\bar{u}, t)$. Since $u=\bar{u}$ lies on a shortest path (due to S1), we have $d(s, t)=$ $d_{0}(s, u)+d_{0}(\bar{u}, t)$. Line 6 implies $d^{\prime} \leq \vec{\delta}(u)+\overleftarrow{\delta}(\bar{u})=d(s, t)$. In fact, this means that the algorithm returns $d^{\prime}=d(s, t)$ since this is already optimal.

Definition 7. For a valid state $z=(P, u, \bar{u})$, the forward direction is said to be blocked if $p \preceq u$. Analogously, the backward direction is blocked if $\bar{u} \preceq p$.

Lemma 11. For a non-final state $z=(P, u, \bar{u})$, at most one direction is blocked.

Proof. Since $z$ is a non-final state, we have $u \prec \bar{u}$, which implies $u \prec p$ or $p \prec \bar{u}$.

Definition 8. The rank $\rho(z)$ of a state $z=(P, u, \bar{u})$ is $|\{x \in P \mid u \preceq x \preceq \bar{u}\}|$.

Lemma 12. From any non-final state $z=(P, u, \bar{u})$, another valid state $z^{+}$is reached at some point such that $\rho\left(z^{+}\right)<\rho(z)$.

Proof. We pick any non-blocked direction-due to Lemma 11, we know that there is at least one such direction. Subsequently, we assume that the forward direction was picked; the backward direction can be dealt with analogously.

We have $u \prec p$ and observe that all prerequisites of Lemma 6 are fulfilled due to Lemmas 9 and 8 . Hence, we can conclude that the edge $(u, v:=\operatorname{succ}(u))$ is not skipped, but relaxed. Thus, since $P$ is a shortest path (S1), $v$ can be reached with the optimal distance due to Lemma $7\left(A_{1}\right)$. The fact that the algorithm terminates (Lemma 3 ) implies that the queue $\vec{Q}$ gets empty at some point, i.e., every element has been deleted from $\vec{Q}$. In particular, we can conclude that $v$ is deleted at some point. Since $v$ has been reached with the optimal distance, it will also be settled with the optimal distance (due to the specification of the decreaseKey operation, which guarantees that tentative distances are never increased). Let $P^{\prime}$ denote the path from $s$ to $v$ in the forward search tree. We set $z^{+}:=\left(P^{+}:=\left.P^{\prime} \circ P\right|_{v \rightarrow t}, v, \bar{u}\right)$. We have $w\left(P^{+}\right)=w\left(P^{\prime}\right)+w\left(\left.P\right|_{v \rightarrow t}\right)=d_{0}(s, v)+d_{0}(v, t)=d_{0}(s, t)(\Rightarrow \mathbf{S} 1)$. S2 is fulfilled since $\left.P^{+}\right|_{v \rightarrow \bar{u}}$ is a subpath of $\left.P\right|_{u \rightarrow \bar{u}}$. S3 holds due to the construction of $P^{+}$. Hence, $z^{+}$is valid. Furthermore, $\rho\left(z^{+}\right)=\rho(z)-1$.

Theorem 5. The highway query algorithm finds a shortest s-t-path.

Proof. From Lemma 12 and the fact that the codomain of the rank function is finite, it follows that eventually a final state is reached, which is equivalent to finding a shortest $s$ - $t$ path due to Lemma 10. 


\section{A.6 Distance Table Optimisation}

To prove the correctness of the distance table optimisation, we introduce the following new lemma and adapt a few definitions and proofs from Section A.5 to the new situation.

Lemma 13. Consider a valid state $z=(P, u, \bar{u})$ with $u \prec s_{L}^{\prime}$. When $u$ 's edges are relaxed, neither the condition in Line 7 a nor the condition in Line 1la is fulfilled.

Proof. Due to Lemma 8, $u$ has been appropriately settled with some key $k$. We distinguish between two cases.

Case 1: $u \prec s_{L}$. From $s_{\ell(u)}=s_{\bar{\ell}(u)} \preceq u \prec s_{L}\left(A_{2}(k, u)\right.$, L2), it follows that $\ell(u)<L$ (U1). Hence, the condition in Line 7a is not fulfilled. Furthermore, we have $s_{\ell} \preceq u \prec s_{L}$ after Line 9 has been executed (Lemma 5: I1). Thus, $\ell<L$, which implies that the condition in Line 11a is not fulfilled as well.

Case 2: $s_{L} \preceq u \prec s_{L}^{\prime}$. First, we show that the condition in Line 7a is not fulfilled. We assume $\ell(u)=L$. (Otherwise, the condition cannot be fulfilled.) Due to $A_{3}(k, u)$, we have $\operatorname{gap}(u)=\infty$. Hence, $\operatorname{gap}^{\prime}=r_{\ell(u)}^{\overrightarrow{1}}(u)=r_{L}(u)=\infty_{1}$ by R1 since $u \notin V_{L}^{\prime}$ (U3). Now, we prove that the condition in Line 11a is not fulfilled. We assume $\ell=L \wedge \ell>\ell(u)$. (Otherwise, the condition cannot be fulfilled.) Due to Line 9, we get gap $=r_{\ell}(u)=r_{L}(u)=\infty_{1}$ (as above).

Definition 6'. A valid state is either a final state (if $u=\bar{u}$ or $s_{L}^{\prime} \preceq u \wedge \bar{u} \preceq t_{L}^{\prime}$ ) or a non-final state (otherwise).

Lemma 10. Getting to a final state is equivalent to finding a shortest s-t-path.

Proof. In the proof of this lemma in Section A.5, we have already dealt with the case $u=\bar{u}$. Now, consider the new case $u \prec \bar{u} \wedge s_{L}^{\prime} \preceq u \wedge \bar{u} \preceq t_{L}^{\prime}$. We show that $s_{L}^{\prime}$ is added to the set $\vec{I}$. Since $s_{L}^{\prime} \preceq u, s_{L}^{\prime}$ has been appropriately settled with some key $k$ (due to Lemma 8). We consider the attempt to relax the edge $\left(s_{L}^{\prime}, v:=\operatorname{succ}\left(s_{L}^{\prime}\right)\right)$ and distinguish between two cases.

Case 1: $s_{L}=s_{L}^{\prime} \cdot \ell=\bar{\ell}(v)$ (I2), $s_{L}=s_{L}^{\prime} \prec v$, and $\bar{\ell}(v) \leq L$ (L1) imply $\ell=\bar{\ell}(v)=L$. Furthermore, $A_{2}\left(k, s_{L}^{\prime}\right)$ and the assumption of Case 1 yield $\ell\left(s_{L}^{\prime}\right)=\bar{\ell}\left(s_{L}^{\prime}\right)<L=\ell$. In addition, gap $=\infty_{2} \neq \infty_{1}$ by I3 (since $s_{\ell}^{\prime} \prec v$ ), the fact that $s_{L}^{\prime} \in V_{L}^{\prime}$ (U4), and R2. Hence, the condition in Line 11a is fulfilled so that $s_{L}^{\prime}$ is added to $\vec{I}$.

Case 2: $s_{L} \prec s_{L}^{\prime}$. By $A_{2}\left(k, s_{L}^{\prime}\right), A_{3}\left(k, s_{L}^{\prime}\right)$, the assumption of Case 2, and $\bar{\ell}\left(s_{L}^{\prime}\right) \leq L$ (L1), we get $\ell\left(s_{L}^{\prime}\right)=\bar{\ell}\left(s_{L}^{\prime}\right)=L$ and $\operatorname{gap}\left(s_{L}^{\prime}\right)=\infty$. Thus, $\operatorname{gap}^{\prime}=r_{L}\left(s_{L}^{\prime}\right)=\infty_{2} \neq \infty_{1}$ (R2). Hence, the condition in Line 7a is fulfilled so that $s_{L}^{\prime}$ is added to $\vec{I}$.

Analogously, we can prove that $t_{L}^{\prime}$ is added to the set $\overleftarrow{I}$. Since $P$ is a highway path (due to Lemma 9), the subpath $\left.P\right|_{s_{L}^{\prime} \rightarrow t_{L}^{\prime}}$ is a path in $G_{L}^{\prime}$ and, thus, $d_{0}\left(s_{L}^{\prime}, t_{L}^{\prime}\right)=d_{L}\left(s_{L}^{\prime}, t_{L}^{\prime}\right)$. Hence, $w(P)=d_{0}\left(s, s_{L}^{\prime}\right)+d_{L}\left(s_{L}^{\prime}, t_{L}^{\prime}\right)+d_{0}\left(t_{L}^{\prime}, t\right)$ is the length of a shortest $s$ - $t$-path and, since the algorithm finds a path with a length $\leq \vec{\delta}\left(s_{L}^{\prime}\right)+d_{L}\left(s_{L}^{\prime}, t_{L}^{\prime}\right)+\overleftarrow{\delta}\left(t_{L}^{\prime}\right)$ and since $\vec{\delta}\left(s_{L}^{\prime}\right)=d_{0}\left(s, s_{L}^{\prime}\right)$ and $\overleftarrow{\delta}\left(t_{L}^{\prime}\right)=d_{0}\left(t_{L}^{\prime}, t\right)$ (due to Lemma 8: $A_{1}$ ), we can conclude that a shortest $s$-t-path is found.

Definition 7'. For a valid state $z=(P, u, \bar{u})$, the forward direction is said to be blocked if $p \preceq u$ or $s_{L}^{\prime} \preceq u$. Analogously, the backward direction is blocked if $\bar{u} \preceq p$ or $\bar{u} \preceq t_{L}^{\prime}$.

Lemma 11. For a non-final state $z=(P, u, \bar{u})$, at most one direction is blocked.

Proof. Since $z$ is a non-final state, we have $u \prec \bar{u}$ and $\left(u \prec s_{L}^{\prime} \vee t_{L}^{\prime} \prec \bar{u}\right)$. To obtain a contradiction, let us assume that both directions are blocked, i.e., $\left(p \preceq u\right.$ or $\left.s_{L}^{\prime} \preceq u\right)$ and 
( $\bar{u} \preceq p$ or $\bar{u} \preceq t_{L}^{\prime}$ ). Consider the case $p \preceq u$ and $\bar{u} \preceq t_{L}^{\prime}$. Hence, $p \preceq u \prec \bar{u} \preceq t_{L}^{\prime}$. Due to M3, we can conclude that $s_{L}^{\prime} \preceq p \preceq u$. Since $s_{L}^{\prime} \preceq u$ and $\bar{u} \preceq t_{L}^{\prime}$, we have a contradiction. The remaining three cases are analogous or straightforward.

Lemma 12. From any non-final state $z=(P, u, \bar{u})$, another valid state $z^{+}$is reached at some point such that $\rho\left(z^{+}\right)<\rho(z)$.

Proof. The proof of this lemma in Section A.5 still works since the added two lines (7a and 11a) have no effect due to Definition 7' and Lemma 13.

\section{B Implementation}

The graph is represented as adjacency array, which is a very space-efficient data structure that allows fast traversal of the graph. There are two arrays, one for the nodes and one for the edges. The edges $(u, v)$ are grouped by the source node $u$ and store only the ID of the target node $v$ and the weight $w(u, v)$. Each node $u$ stores the index of its first outgoing edge in the edge array. In order to allow a search in the backward graph, we have to store an edge $(u, v)$ also as backward edge $(v, u)$ in the edge group of node $v$. In order to distinguish between forward and backward edges, each edge has a forward and a backward flag. By this means, we can also store two-way edges $\{u, v\}$ (which make up the large majority of all edges in a real-world road network) in a space-efficient way: we keep only one copy of $(u, v)$ and one copy of $(v, u)$, in each case setting both direction flags.

The basic adjacency array has to be extended in order to incorporate the level data that is specific to highway hierarchies. In addition to the index of the first outgoing edge, each node $u$ stores its level- 0 neighbourhood radius $r_{0}(u)$. Moreover, for each node $u$, all outgoing edges $(u, v)$ are grouped by their level $\ell(u, v)$. Between the node and the edge array, we insert another layer: for each node $u$ and each level $\ell>0$ that $u$ belongs to, there is a level node $u_{\ell}$ that stores the radius $r_{\ell}(u)$ and the index of the first outgoing edge $(u, v)$ in level $\ell$. All level nodes are stored in a single array. Each node $u$ keeps the index of the level node $u_{1}$. Figure 16 illustrates the graph representation.

nodes

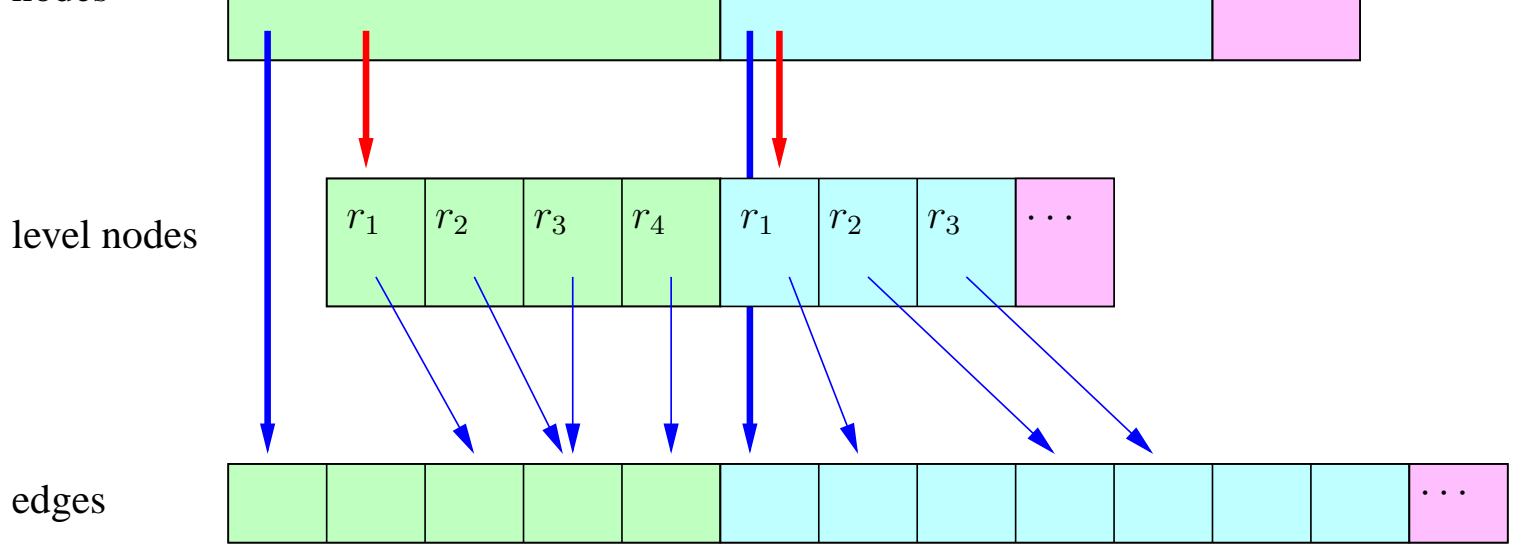

Fig. 16. An adjacency array, extended by a level-node layer.

To obtain a robust implementation, we include extensive consistency checks in assertions and perform experiments that are checked against reference implementations, i.e., queries are checked against Dijkstra's algorithm and the fast preprocessing algorithm is checked against a naive implementation. 


\section{Experiments}

In addition to the experiments presented in Section 6.3, we have considered many more combinations of neighbourhood size, contraction rate, and number of levels. The results are given in Table 5 and 6.

Table 5. Preprocessing and query performance for the European road network depending on the contraction rate $c$ and the neighbourhood size $H$. We do not use a distance table, but repeat the construction process until the topmost level is empty or the hierarchy consists of 15 levels.

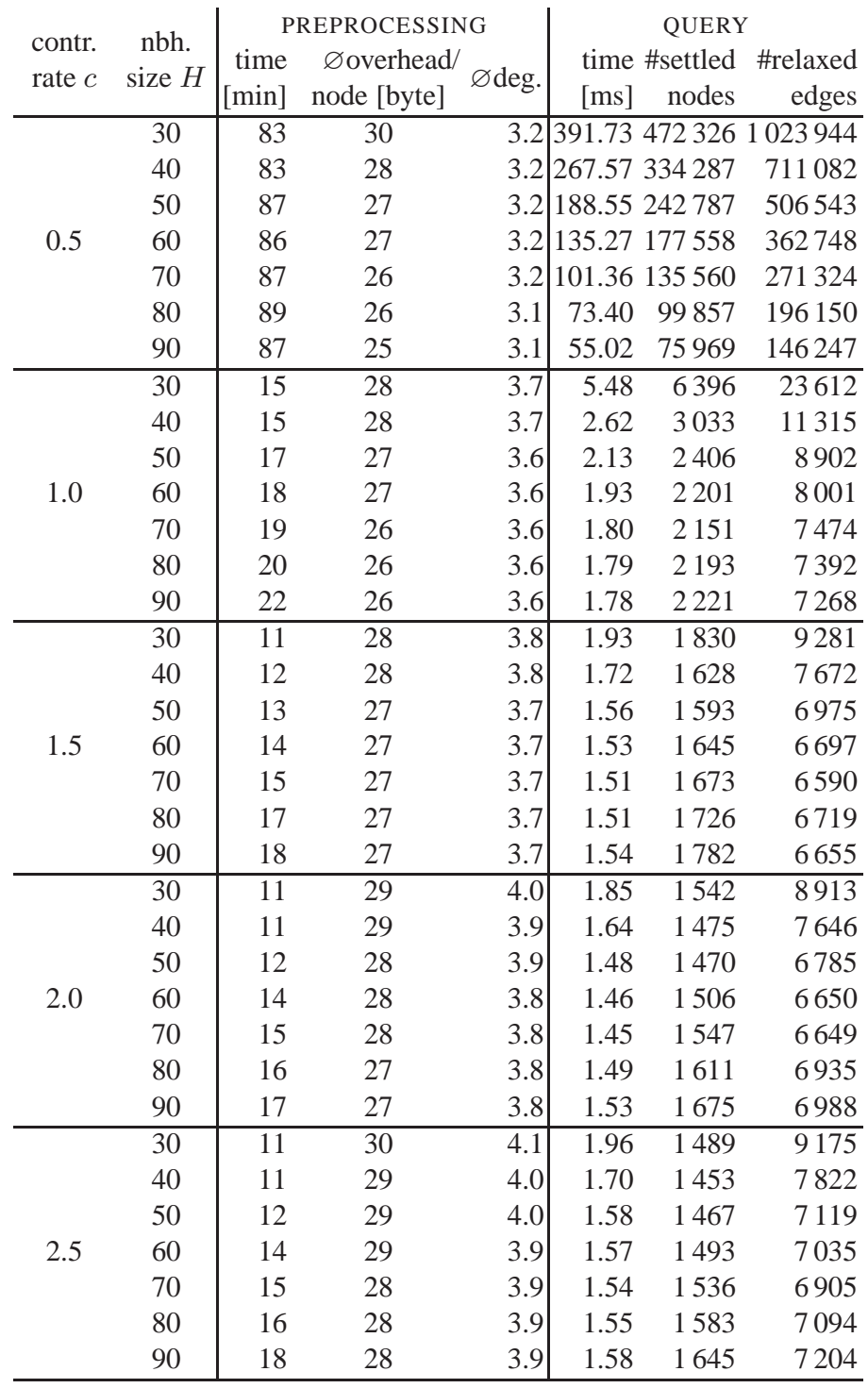


Table 6. Preprocessing and query performance for the European road network depending on the number of levels and the neighbourhood size $H$. In the topmost level, a distance table is used.

\begin{tabular}{|c|c|c|c|c|c|c|c|}
\hline \multirow{3}{*}{ \#levels } & \multirow{3}{*}{$\begin{array}{c}\text { nbh. } \\
\text { size } H\end{array}$} & \multicolumn{3}{|c|}{ PREPROCESSING } & \multicolumn{3}{|c|}{ QUERY } \\
\hline & & time & $\varnothing$ overhead/ & & time & \#settled & relaxed \\
\hline & & [min] & node [byte] & & [ms] & nodes & edges \\
\hline \multirow{6}{*}{5} & 40 & 14 & 60 & 3.9 & 0.67 & 691 & 2398 \\
\hline & 50 & 13 & 40 & 3.9 & 0.77 & 818 & 2892 \\
\hline & 60 & 14 & 32 & 3.8 & 0.87 & 938 & 3361 \\
\hline & 70 & 15 & 30 & 3.8 & 0.96 & 1058 & 3837 \\
\hline & 80 & 16 & 28 & 3.8 & 1.05 & 1165 & 4278 \\
\hline & 90 & 17 & 28 & 3.8 & 1.13 & 1269 & 4697 \\
\hline \multirow{7}{*}{6} & 30 & 12 & 48 & 4.0 & 0.75 & 709 & 2531 \\
\hline & 40 & 11 & 33 & 3.9 & 0.87 & 867 & 3171 \\
\hline & 50 & 12 & 29 & 3.9 & 0.99 & 1015 & 3759 \\
\hline & 60 & 13 & 28 & 3.8 & 1.10 & 1157 & 4299 \\
\hline & 70 & 15 & 28 & 3.8 & 1.21 & 1292 & 4837 \\
\hline & 80 & 16 & 28 & 3.8 & 1.30 & 1414 & 5311 \\
\hline & 90 & 17 & 27 & 3.8 & 1.40 & 1521 & 5817 \\
\hline \multirow{7}{*}{7} & 30 & 10 & 34 & 4.0 & 0.93 & 852 & 3195 \\
\hline & 40 & 11 & 29 & 3.9 & 1.07 & 1025 & 3894 \\
\hline & 50 & 12 & 28 & 3.9 & 1.20 & 1187 & 4538 \\
\hline & 60 & 13 & 28 & 3.8 & 1.32 & 1344 & 5166 \\
\hline & 70 & 15 & 28 & 3.8 & 1.39 & 1462 & 5689 \\
\hline & 80 & 16 & 27 & 3.8 & 1.47 & 1578 & 6179 \\
\hline & 90 & 18 & 27 & 3.8 & 1.53 & 1668 & 6661 \\
\hline \multirow{7}{*}{8} & 30 & 10 & 30 & 4.0 & 1.14 & 991 & 3853 \\
\hline & 40 & 11 & 29 & 3.9 & 1.27 & 1171 & 4624 \\
\hline & 50 & 12 & 28 & 3.9 & 1.36 & 1321 & 5283 \\
\hline & 60 & 14 & 28 & 3.8 & 1.43 & 1455 & 5887 \\
\hline & 70 & 15 & 28 & 3.8 & 1.46 & 1546 & 6338 \\
\hline & 80 & 16 & 27 & 3.8 & 1.48 & 1611 & 6935 \\
\hline & 90 & 18 & 27 & 3.8 & 1.53 & 1675 & 6988 \\
\hline \multirow{5}{*}{9} & 30 & 10 & 30 & 4.0 & 1.35 & 1123 & 4532 \\
\hline & 40 & 11 & 29 & 3.9 & 1.45 & 1289 & 5338 \\
\hline & 50 & 12 & 28 & 3.9 & 1.48 & 1417 & 5931 \\
\hline & 60 & 14 & 28 & 3.8 & 1.47 & 1506 & 6429 \\
\hline & 70 & 15 & 28 & 3.8 & 1.46 & 1547 & 6649 \\
\hline \multirow{4}{*}{10} & 30 & 10 & 29 & 4.0 & 1.54 & 1241 & 5214 \\
\hline & 40 & 11 & 29 & 3.9 & 1.57 & 1380 & 6012 \\
\hline & 50 & 12 & 28 & 3.9 & 1.51 & 1468 & 6470 \\
\hline & 60 & 14 & 28 & 3.8 & 1.46 & 1506 & 6650 \\
\hline \multirow{3}{*}{11} & 30 & 10 & 29 & 4.0 & 1.67 & 1326 & 5847 \\
\hline & 40 & 11 & 29 & 3.9 & 1.65 & 1445 & 6627 \\
\hline & 50 & 13 & 28 & 3.9 & 1.49 & 1470 & 6785 \\
\hline
\end{tabular}

